\title{
Evaluation by tunneling effect for the temperature-dependent electric conductivity of polymer-carbon fiber composites with visco-elastic properties
}

\author{
Rong Zhang, Yuezhen Bin, Ru Chen and Masaru Matsuo
}

The conductivity of composites with a carbon fiber (CF) content slightly higher than the percolation threshold was measured at increasing temperatures up to $-10^{\circ} \mathrm{C}$. The tunneling effect was theoretically calculated using a rigorous nonparabolic potential barrier. The tunneling barrier width $D$ and the surface area $A$ were determined. For a polyethylene (PE) matrix, good agreement between theoretical and experimental results was obtained using $D=1.00 \mathrm{~nm}$ and $A=1.35$ to $1.68 \mathrm{~nm}^{2}$ at a 30 -vol\% CF content and using $D=1.30 \mathrm{~nm}$ and $A=1.25$ to $1.28 \mathrm{~nm}^{2}$ at a $25-\mathrm{vol} \%$ CF content. That is, almost perfect agreement between experiment and theory was obtained by adjusting the parameters except over the temperature ranges in which the $\beta$ and $\gamma$ relaxation peaks appeared. Dynamic loss modulus and positron annihilation measurements were also conducted. However, a theoretical analysis that was derived using a parabolic potential barrier produced inconsistent results. That is, the tunneling barrier width $D$ was less than the $c$-axis length of a PE crystal unit, and the surface area $A$ was considerably less than the $a-b$ plane area of a PE crystal unit. Polymer Journal (2013) 45, 1120-1134; doi:10.1038/pj.2013.40; published online 10 April 2013

Keywords: conductive polymer composite; electron transport; fluctuation-induced tunneling conduction; nonparabolic potential barrier width; visco-elastic property

\section{INTRODUCTION}

It is well-known that the conductivity of composite systems, such as polymer-carbon nanotube composites, polymer-carbon fiber (CF) composites, and polymer-carbon black composites, increases with temperature up to $0{ }^{\circ} \mathrm{C}$ and then decreases upon further increasing the temperature. ${ }^{1-12}$ The conductivity increase up to $0{ }^{\circ} \mathrm{C}$ by the enhancement of electron transport has been attributed to two mechanisms, electron hopping ${ }^{13-17}$ and electron tunneling. ${ }^{2-5,18-22}$

Electron hopping is thought to occur by two mechanisms. The first mechanism predominates at high temperatures at which there is sufficient excess thermal energy to excite the electrons into the conduction band, and the conductivity can be evaluated using Arrhenius plots. The second mechanism is known as variable range hopping (VRH) and has been theoretically modeled by Mott and Davis $^{13}$ at low temperatures, the excited electrons lose their ability to jump into the conduction band and instead attempt to find a state with similar energy to their own by hopping beyond their nearest neighbors to more distant sites, corresponding to a greater selection of possible electron energy levels.

Two types of electron tunneling have been observed. The first type is the electric tunneling effect, which occurs between similar electrodes that are separated by a thin insulating film, and was discovered by Simmons ${ }^{18}$ the current given by the mean barrier height above the Fermi level of the negatively biased electrode can be approximated by the product of the electron charge and the voltage across the film at absolute temperature. The second type is fluctuation-induced tunneling conduction in disordered materials, which was discovered by Sheng et al. ${ }^{19,20}$ The authors modeled the temperature-dependent conductivity using a fluctuation probability function when the thermal fluctuation field was stronger than the applied field. That is, the logarithm of the Fermi-Dirac function was approximated as $-E$ for $E<0$ and 0 for $E>0$ (where $E$ denotes the electron energy in the tunneling direction) to facilitate numerical calculation. Sheng's theory has been appropriated in many studies, ${ }^{2-5,7,20-22}$ in conjunction with a parabolic potential barrier, to estimate the conductivity of polymer semi-conductive and conductive filler systems as a function of the absolute temperature $T$, that is,

$$
\sigma(T) \propto \exp \left[-\frac{T_{1}}{T+T_{0}}\right]
$$

where $T_{0}$ is a parameter that may be interpreted as a limit temperature associated with the thermal fluctuations above $T$, and 
$T_{1}$ is related to the shape of the energy barrier to tunneling. However, the conductivity given by Equation (1) is only valid for a parabolic potential barrier.

In this study, the temperature dependence of electron transport is theoretically and experimentally investigated for ultra-high molecular weight polyethylene (UHMWPE)-CF composites using a nonparabolic function for the potential barrier in Sheng's general theory. ${ }^{19}$ Simmons ${ }^{18}$ and Sheng ${ }^{19}$ showed that the potential barrier, which is represented by an additional parameter in the image-force correction, can be modeled by a wide variety of barrier shapes ranging from approximately rectangular to approximately parabolic to study the effect of voltage fluctuations. To validate the fitting procedure between the experimental and theoretical results in this study, numerical calculations were carried out for UHMWPE-CF composites; these materials were chosen because polyethylene (PE) is a typical insulator and has the lowest conductivity of many types of insulating polymers.

The most controvertible issue in the fitting procedure is that Equation (1) is only valid for a parabolic potential barrier. The results and discussion section shows that using this analysis for a composite with a $30-\mathrm{vol} \%$ CF content results in serious inconsistencies: the width of the potential barrier is less than the length of the $c$-axis of the PE crystal unit, and the surface area over which most of tunneling occurs is considerably less than the $a-b$ plane area of the PE crystal unit. These major contradictions can be resolved by using a nonparabolic potential barrier (which is given by (11) later in the paper) and an estimated barrier width of $1.00 \mathrm{~nm}$ below $0{ }^{\circ} \mathrm{C}$, which is a well-known rigorous value for metal-metal and metalsemiconductor junctions. ${ }^{8,23}$

To further validate the application of the fluctuation-induced tunneling effect in polymer-filler composite systems using a nonparabolic potential barrier, we investigate another significant problem in using VRH to model insulating polymer semi-conductive filler (such as CF and carbon nanotube composites) composites. Mott's VRH analysis is completely appropriate for semiconducting phases such as $\mathrm{TiO}_{2}$ doped by $\mathrm{Co}, \mathrm{Cr}, \mathrm{Cd}, \mathrm{Ce}$ and $\mathrm{Fe},{ }^{14}$ and the widespread application of VRH to doped semiconductors, amorphous conductors, glasses and conductive and semi-conductive polymers has also been successful. ${ }^{14-17,24-27}$ However, composite systems consisting of conductive or semi-conductive fillers embedded in insulating polymers do not lie within Mott's VRH framework. This paper mainly focuses on two features in modeling electron transport: (1) the limits of application for the VRH theory and (2) suitable applications of the fluctuation-induced tunneling effect using a nonparabolic potential barrier.

\section{EXPERIMENTAL PROCEDURE}

\section{Materials and sample preparation}

In this study, UHMWPE (Mitsui Chemicals, Hizex) with a viscosity average molecular weight of $6.3 \times 10^{6}$ was used. Short CFs carbonized beyond $2000^{\circ} \mathrm{C}$, with an average filament diameter of $\sim 7 \mu \mathrm{m}$ and an average length of $\sim 40 \mu \mathrm{m}$, were supplied by Teijin Co., Tokyo, Japan. The UHMWPE-CF composites were prepared from solution by a gelation/crystallization method using $p$-xylene as a solvent. The CF volume fractions used in the UHMWPE composites were $15,20,25,28,30,35,45,90$ and 99 vol\%. The UHMWPE

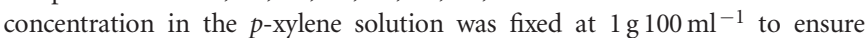
uniform mixing. The CFs were uniformly dispersed into the UHMWPE solutions by alternating ultrasonic dispersion and stirring for over $2 \mathrm{~h}$ at room temperature. The UHMWPE powder was then added into a solvent containing the $\mathrm{CFs}$, and the mixture was stirred for $30 \mathrm{~min}$ at $140^{\circ} \mathrm{C}$ under a nitrogen atmosphere. The hot homogeneous solution was quenched by pouring it into a glass tray at room temperature. The $p$-xylene was allowed to evaporate from the gel under ambient conditions, and the resulting film was completely dried at $60^{\circ} \mathrm{C}$. The dried films were pressed under $2 \mathrm{MPa}$ at $180^{\circ} \mathrm{C}$ for $10 \mathrm{~min}$, followed by cooling using one of two methods. In the first method, the film was cooled to room temperature at the same pressure, and in the second method, the film was quenched in liquid nitrogen immediately after removing the melted sample from the hot-pressed machine. The composites with 90 and 99 vol\% CF content were pressed again under $5 \mathrm{MPa}$ at room temperature for $20 \mathrm{~min}$ to form a uniform film by aggregating the short CFs without causing brittle damage. For the composites with 90 and 99 vol\% CF content, 10 and 1 vol\% UHMWPE were used as adhesives to form film-like stable composites with dense contacts between adjacent CF surfaces. Both surfaces of all the resulting molded films were polished with sandpaper to remove as much UHMWPE as possible from the surfaces.

\section{Measurements}

The electrical conductivity of the samples was measured using a two-terminal method, in which the two ends of the specimen were clamped between copper metal jaws in a hot oven. The test specimens were prepared by cutting the composite into 30-mm-long and 10-mm-wide strips; the sample length between the copper jaws was $10 \mathrm{~mm}$, and the thickness of the samples was $\sim 1.0 \mathrm{~mm}$. The detailed measurement method has been described elsewhere. ${ }^{28,29}$ The electrical conductivity was measured under serial voltages in a home-made circuit. For the composites with up to 99 vol\% CF content, the voltage was supplied by a DC power supply that was adjusted between 0.4 and $18 \mathrm{~V}$, and the conductivity was measured with a digital multimeter (Advantest R6441A Digital Multimeter, Advantest, Tokyo, Japan); the same measurement was performed for the long CFs. For temperatures ranging between -146 and $15^{\circ} \mathrm{C}$, the conductivity was obtained from current measurements using the aforementioned digital multimeter. The temperature in the sample chamber was controlled by a commercial program, and liquid nitrogen was used to decrease the temperature. The temperature was first decreased to $-146^{\circ} \mathrm{C}$, at which it was maintained for $10 \mathrm{~min}$, after which the temperature was increased to $15^{\circ} \mathrm{C}$ at a rate of $5^{\circ} \mathrm{C} \mathrm{min}-1$. No thermal expansion was observed in the measurements.

The thermal properties of the annealed and quenched specimens of composites with 25 and 30 vol\% CF content were measured by differential scanning calorimetry from 20 to $180^{\circ} \mathrm{C}$ using a differential scanning calorimetry-204 (NETZSCH) at a heating rate of $10^{\circ} \mathrm{Cmin}^{-1}$ in a $\mathrm{N}_{2}$ atmosphere. The weight of the samples, which were sealed in aluminum pans, was $\sim 9 \mathrm{mg}$. The crystallinities of the UHMWPE matrix for the annealed and quenched specimens were $51.4 \%$ and $34.6 \%$, respectively; these values were calculated using the heat of fusion of each specimen, the heat of fusion of $100 \% \mathrm{PE}\left(280.5 \mathrm{Jg}^{-1}\right)$ and the volume content of the UHMWPE. The crystallinity of the annealed specimen with a $25-\mathrm{vol} \%$ CF content was $\sim 52.3 \%$. The differential scanning calorimetry curves are not shown here for brevity.

The temperature-dependent dynamic tensile modulus was measured for the composites with a 30-vol\% CF content using a visco-elastic spectrometer from Iwamoto Machine Co. Ltd (Kyoto, Japan) at a fixed frequency of $10 \mathrm{~Hz}$ over temperatures ranging from $-146^{\circ} \mathrm{C}$ to room temperature (which was $\sim 25^{\circ} \mathrm{C}$ ). The specimens used in this experiment were cut into samples with a length of $40 \mathrm{~mm}$ and a width of $2 \mathrm{~mm}$ and were clamped at the ends over a length of $10 \mathrm{~mm}$. The initial load at $-146^{\circ} \mathrm{C}$ was $2.15 \mathrm{MPa}$, and the applied external strain was $0.01 \mathrm{~mm}$. The visco-elastic spectrometer could automatically decrease the load as the temperature was increased to select the best conditions for measuring the storage and loss moduli as a function of temperature.

The scanning electron microscope studies were performed using a QUANTA 450. The cross-sections of the composite film were prepared by freezing the film with liquid nitrogen, followed by cracking the film by hand; gold was then coated onto the film surface to view the fractured surface.

\section{Characterization}

Before investigating the electrical properties of the UHMWPE-CF composites, a supplementary experiment was performed to determine the most suitable CF concentration. Figure 1a shows that the conductivity $(\sigma)$ of the composites 

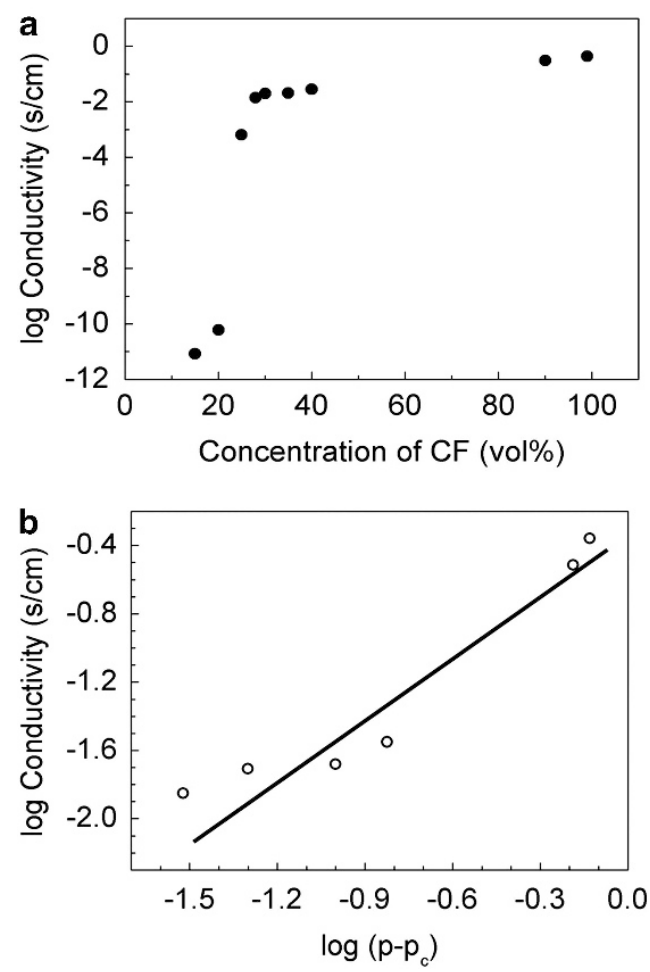

Figure 1 Conductivity of ultra-high molecular weight polyethylene (UHMWPE)-carbon fiber (CF) composites versus CF concentration: (a) logarithmic plots of conductivity versus CF concentration for UHMWPE-CF composites at room temperature; (b) logarithmic plots of conductivity versus $\log \left(p-p_{c}\right)$ calculated using Equation (2).

increased with the CF content. The conductivity jumped drastically above 20 vol\% CF and leveled-off with further increases in the CF content, indicating a percolation phenomenon. In classical percolation theory, the conductivity is related to the volume content as follows:

$$
\sigma \propto\left(p-p_{c}\right)^{t} \text { for } p>p_{c}
$$

where $p$ is the volume content and $p_{c}$ is the critical volume content at the percolation threshold, which was experimentally determined to be $25 \mathrm{vol} \% \mathrm{CF}$ in this study. The exponent $t$ characterizes the relationship between $\sigma$ and $p$ and had a value of 1.53 , indicating a three-dimensional percolation system..$^{30,31}$ These results were used to select composites with a 30 -vol\% CF content and a 25-vol\% CF content as specimens to ensure overlap between neighboring CFs. The 30 vol\% CF content was slightly higher than the critical 25 vol\% CF content to ensure sufficient overlap between neighboring CFs; the sample with the $25 \mathrm{vol} \%$ CF content was selected because it was close to the percolation threshold. In preliminary experiments, the temperature-dependent conductivity could not be measured (for comparison with the theoretical results) for the composite with $<25 \mathrm{vol} \%$ CF content because the electrical current was extremely low. Consequently, the composites with 25, 30 and $99 \mathrm{vol} \%$ CF content were selected as test specimens.

In another supplementary experiment, scanning electron microscope images (see Figures $2 \mathrm{a}$ and $\mathrm{b}$ ) were obtained for cross-sections of composites with $99 \mathrm{vol} \%$ and $30 \mathrm{vol} \%$ CF content, respectively, to probe the arrangement of the CFs in the UHMWPE medium. The image in Figure $2 \mathrm{~b}$ for the $30 \mathrm{vol} \%$ CF composite with $34.6 \%$ crystallinity $(X \mathrm{c})$ was selected because this image was similar to that of the composite with $51.4 \%$ crystallinity. Figure 2a shows dense overlapping between adjacent CFs for the composite with a 99 -vol\% CF content. This image reveals the contact between adjacent CF surfaces through small air void gaps and/or through very thin scattered UHMWPE fragments on adjacent CF surfaces. However, Figure $2 \mathrm{~b}$ shows a composite with a $30 \mathrm{vol} \%$
CF content for which some parts of a CF were covered by the UHMWPE fibrous structure, indicating that the UHMWPE chains tended to crystallize on the CF surfaces, as has been previously reported for MWCNT-UHMWPE composites. ${ }^{28}$

\section{RESULTS AND DISCUSSION}

First, the temperature-dependent conductivities were measured for long and short CFs coated by very thin UHMWPE fractures to investigate the mechanisms of (1) electron hopping and (2) electron tunneling. Direct measurement of the conductivity of the long CFs directly probes characteristics along the CF chains, whereas measuring the conductivity of the short CFs probes the lateral contact between adjacent CF surfaces (which are perpendicular to the chain axes). ${ }^{12} \mathrm{~A}$ detailed comparison between the long CFs and the 99 vol\% $\mathrm{CF}$ content film showed that the conductivity of the two composites with the 30 vol\% CF content but different crystallinities should be analyzed in terms of the tunneling effect with thermal fluctuations and a nonparabolic potential barrier to obtain conclusive results. In this paper, conclusive evidence for the temperature-dependent conductivity of the composites is presented using the results for three composites with 25,30 and 99 vol\% CF content.

\section{Electron hopping}

In the classical literature, ${ }^{14,32-34}$ electron hopping in semi-conductive materials are classified into high and low temperature categories, as previously discussed. At high temperatures, there is sufficient excess thermal energy to excite electrons into the conduction band. The conductivity can be described by a thermally activated process, which can be expressed by an Arrhenius relation as follows:

$$
\sigma=\sigma_{0} \exp \left(-\frac{\Delta E}{k T}\right)
$$

where $\Delta E$ denotes the activation energy for conduction, $k$ denotes Boltzmann's constant, $T$ denotes the absolute temperature and $\sigma_{0}$ denotes a constant, which is equal to the conductivity at zero Kelvin.

At low temperatures, the conductivity obeys a $T^{-1 / 4}$ power law corresponding to the three-dimensional VRH of the conductivity between localized states. In VRH, electron hopping occurs predominantly beyond nearest neighbors. ${ }^{13,14,17,27}$ The VRH model for the conductivity is given by

$$
\sigma=\sigma_{0} T^{-1 / 2} \exp \left[-\left(\frac{T_{0}}{T}\right)^{1 / 4}\right]
$$

where

$$
\sigma_{0}=\left[\frac{N\left(E_{\mathrm{F}}\right)}{2 \pi \alpha k T}\right]^{-1 / 2}\left(\frac{3 e^{4} v^{2} \phi_{0}}{2}\right)^{-1 / 2}
$$

and

$$
T_{0}=\frac{18 \alpha^{3}}{k N\left(E_{\mathrm{F}}\right)}
$$

where $N\left(E_{\mathrm{F}}\right)$ is the density of the localized states at the Fermi level, $\alpha$ is the coefficient of the exponential decay of the wave function of the localized states, $v$ is the typical phonon frequency and $\phi_{0}$ is the overlapping integral, which is on the order of unity. 


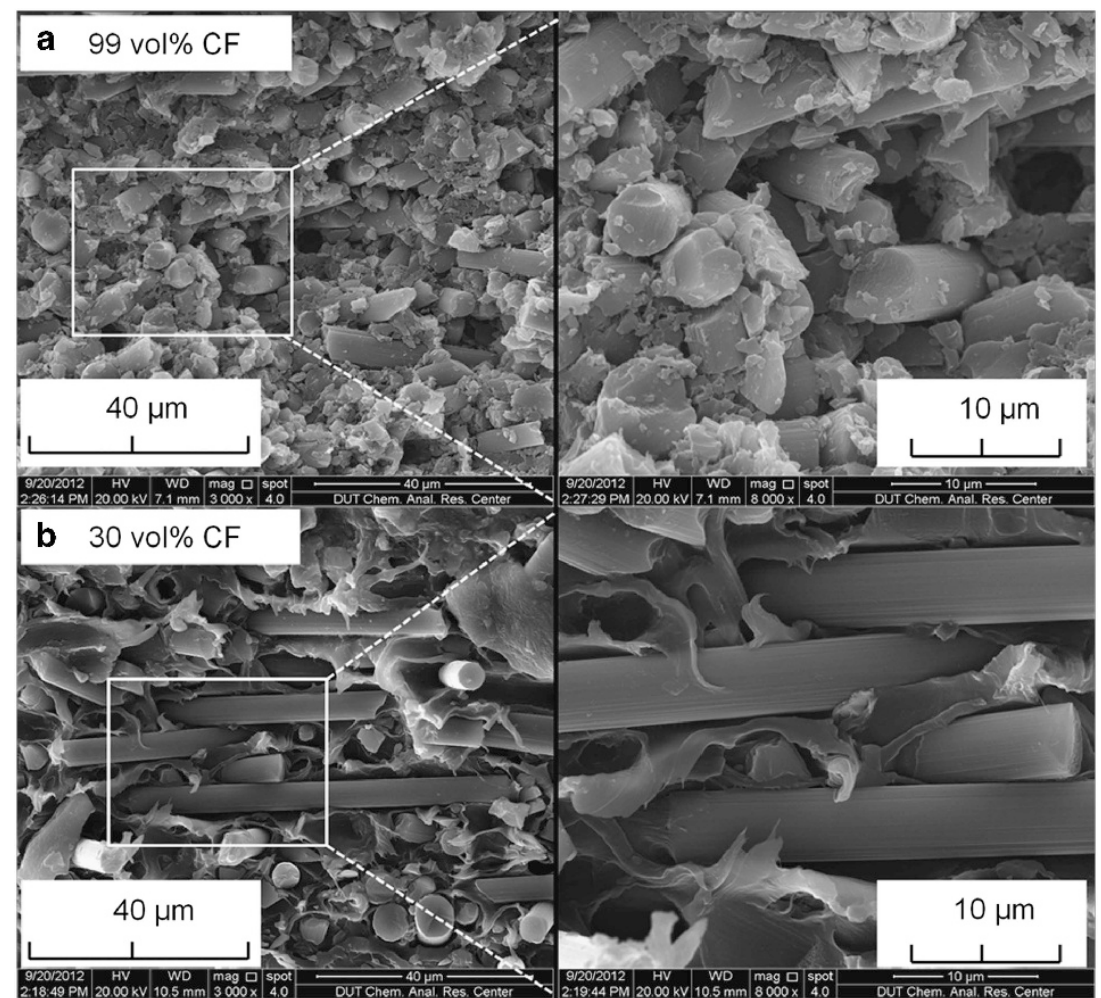

Figure 2 Scanning electron microscope images of the cross-sectional area of the ultra-high molecular weight polyethylene (UHMWPE)-carbon fiber (CF) composites and corresponding magnified images: (a) composite with a 99 vol\% CF content and (b) $X \mathrm{C}=34.6 \%$ for a composite with 30 vol\% CF content.

The hopping distance $R$ and the average hopping energy $W$ can be calculated using the following equations:

$$
\begin{aligned}
& R=\left[\frac{9}{8 \pi \alpha T N\left(E_{\mathrm{F}}\right)}\right]^{1 / 4} \\
& W=\frac{9}{4 \pi R^{3} N\left(E_{\mathrm{F}}\right)}
\end{aligned}
$$

The aforementioned analysis was developed by Okutan et al. ${ }^{14}$ for semiconducting Co-doped $\mathrm{TiO}_{2}$ and by Khan et al. ${ }^{17}$ for multi-wall carbon nanotubes (MWNTs).

In this study, Mott's VRH theory ${ }^{13}$ was applied to both the long and short CFs. Figure 3a shows the conductivity $(\sigma)$ as a function of temperature $(T)$ for the long CFs, as measured by a digital meter, and Figure $3 \mathrm{~b}$ shows $\sigma$ versus $T$ for the short CFs, as measured by the voltages indicated. The conductivity of the long CFs increased with temperature, whereas the conductivity of the short aggregated $\mathrm{CFs}$ attained a maximum at approximately $-80^{\circ} \mathrm{C}$ and then decreased.

The increase in the conductivity of the long CFs with temperature is a typical phenomenon and reflects the acceleration in the electron hopping from a valence band (a filled band) to a conduction band or the motion of positive holes to empty 'seats' in the valence band, which is indicative of a p-type semiconductor. ${ }^{35}$ To validate the applicability of these concepts to polymer-filler composites, $\ln \sigma$ versus $1 / T$ was first plotted (see Figure $3 \mathrm{c}$ ); the plot could be approximated by two intersecting straight lines. Klimenko et al. ${ }^{36}$ have reported the same trend for $\mathrm{CF}$ carbonized at $2200^{\circ} \mathrm{C}$. The intersection point of the two lines in Figure $3 \mathrm{c}$ was approximately $-50{ }^{\circ} \mathrm{C}$, reflecting the two conductive mechanisms. The current above $-50{ }^{\circ} \mathrm{C}$ could be attributed to electrons that were excited into the conduction band by sufficient excess thermal energy. However, the activation energy calculated from Equation (3) was $\sim 2.4 \times 10^{-3} \mathrm{eV}$. This value was similar to the activation energy for networkednanographite $^{37}\left(4.0 \times 10^{-3} \mathrm{eV}\right)$ and one-order lower than the activation energy of an inorganic material, Co-doped $\mathrm{TiO}_{2}$ $\left(3.2 \times 10^{-2} \mathrm{eV}\right) .{ }^{14}$ Note that the activation energies above $-50{ }^{\circ} \mathrm{C}$ were not in the regime where the excess thermal energy was sufficient to excite electrons into the conduction band because $2.4 \times 10^{-3} \mathrm{eV}$ is too low to ensure electron excitation compared with the energy bands for intrinsic semiconductors and acceptor levels for impurity semiconductors by doping. Examples of reported activation energies are $1.04 \times 10^{-2} \mathrm{eV}$ for $\mathrm{B}-\mathrm{Ge}, 1.02 \times 10^{-2} \mathrm{eV}$ for Al-Ge, and $1.08 \times 10^{-2} \mathrm{eV}$ for Ga-Ge. ${ }^{38}$ The activation energy of $5.6 \times 10^{-5} \mathrm{eV}$ below $-50^{\circ} \mathrm{C}$ was unreasonable. The low activation energies at high and low temperatures using Equation (3) were meaningless for the long CFs.

Motivated by the VRH theory, the aforementioned contradiction was resolved by plotting $\ln \sigma T^{1 / 2}$ versus $T^{-1 / 4}$ (see Figure 3e). The calculated plot was classified as two regions that intersected at approximately $-50{ }^{\circ} \mathrm{C}$; however, the difference between the two slopes was small enough to be neglected. The plots were linear within the experimental error. The value of $T_{0}$ at $4300 \mathrm{~K}$ was estimated from the slope of the straight line. The value of $N\left(E_{\mathrm{F}}\right)$ was calculated to be $4.77 \times 10^{22} \mathrm{eV}^{-1} \mathrm{~cm}^{-3}$, assuming a value of $10^{-7} \mathrm{~cm}$ for $1 / \alpha$ from Yakuphanoglu et al. ${ }^{27}$ Figure $3 \mathrm{~g}$ shows the hopping distance $R$ (as a solid curve) and the average hopping energy $W$ (as a dashed curve) as a function of $T$. The value of $R$ decreased with temperature, whereas the value of $W$ increased with the temperature, as has been reported for many materials. The temperature-dependent $R$ values were greater than those of $\mathrm{MWNTs}^{17}$ and $\mathrm{La}_{0.67} \mathrm{Ca}_{0.33} \mathrm{MnO}_{3}$ perovskite 

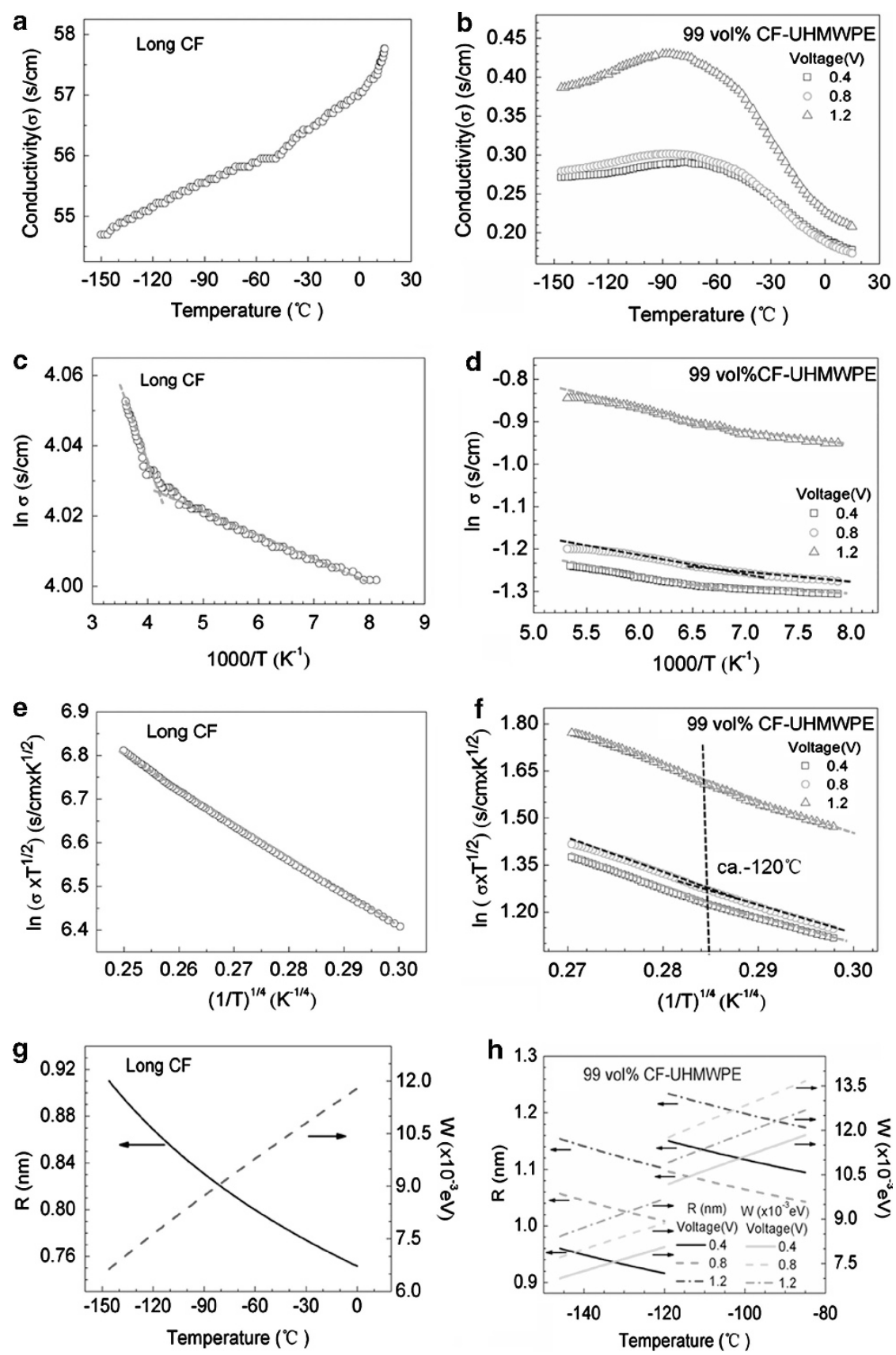

Figure 3 (a) Conductivity of long carbon fibers (CFs) and a composite with 99 vol\% CF-UHMWPE (ultra-high molecular weight polyethylene) versus temperature ( $T$ ); plots of the natural logarithm of the conductivity In $\sigma$ versus $1000 / T$ for (b) long CFs and (c) a composite with a $99-$ vol\% CF content; plots of $I n \sigma T^{1 / 2}$ versus $T^{-1 / 4}$ for (d) long CFs and (e) a composite with a 99-vol\% CF content; plots of $R$ versus $T$ and $W$ versus $T$ for (g) long CFs and (h) composites with a $99-$ vol\% CF content. A full color version of this figure is available at Polymer Journal online.

magnetite. ${ }^{24}$ This result may be attributed to the slightly higher conductivity of long CFs compared with those of MWNTs and $\mathrm{La}_{0.67} \mathrm{Ca}_{0.33} \mathrm{MnO}_{3}$ perovskite magnetite. The temperature-dependent $W$ values were almost equal to those for MWNTs but were much lower than those for $\mathrm{La}_{0.67} \mathrm{Ca}_{0.33} \mathrm{MnO}_{3}$ perovskite magnetite. These results are expected because CFs and MWNTs have the same graphene structure, whereas $\mathrm{La}_{0.67} \mathrm{Ca}_{0.33} \mathrm{MnO}_{3}$ perovskite magnetite requires a large average hopping energy to ensure current flow. Nevertheless, the linearity of the $\ln \sigma T^{1 / 2}$ versus $T^{-1 / 4}$ plots for the long CFs validated the $\mathrm{VRH}$ theory over a wide temperature range from -146 to $0^{\circ} \mathrm{C}$. $^{7,24,27}$
Figure $3 \mathrm{~b}$ shows that the conductivity of the molded short CF film was $\sim 2-3$ orders lower than the conductivity of the long CFs (see Figure $3 \mathrm{a}$ ). The conductivity at $1.2 \mathrm{~V}$ was higher than that at 0.8 and $0.4 \mathrm{~V}$. The dependence of the applied field on the conductivity was indicative of a deviation from the well-known Ohmic law. The conductivity for the voltages indicated increased slightly with the temperature up to $-80^{\circ} \mathrm{C}$. However, the conductivity decreased upon increasing the temperature above $-80^{\circ} \mathrm{C}$. The difference in the behavior of the conductivity below and above approximately $-80^{\circ} \mathrm{C}$ could be attributed to the thermal expansion of small air voids ${ }^{39,40}$ in the gaps among short neighboring CFs. 
Figure $3 \mathrm{~d}$ shows the corresponding $\ln \sigma$ versus $1 / T$ plot. This plot consisted of two intersecting straight lines with slightly different slopes. The calculated $\Delta E$ values in the high temperature regime (that is, above approximately -120 to $-128^{\circ} \mathrm{C}$ ) were approximately $3.5 \times 10^{-3} \mathrm{eV}$ at $0.4 \mathrm{~V}, 3.5 \times 10^{-3} \mathrm{eV}$ at $0.8 \mathrm{~V}$, and $4.7 \times 10^{-3} \mathrm{eV}$ at $1.2 \mathrm{~V}$, and the calculated $\Delta E$ values in the low temperature regime (that is, below approximately -120 to $-128^{\circ} \mathrm{C}$ ) and $1.2 \times 10^{-3} \mathrm{eV}$ at $0.4 \mathrm{~V}, 2.2 \times 10^{-3} \mathrm{eV}$ at $0.8 \mathrm{~V}$ and $3.0 \times 10^{-3} \mathrm{eV}$ at $1.2 \mathrm{~V}$. The extremely low $\Delta E$ values invalidated the use of Arrhenius plots for evaluating the conductivity of the short CFs, similar to the case of the long CFs.

The data were further analyzed by the VRH theory by plotting $\ln \sigma T^{1 / 2}$ versus $T^{-1 / 4}$ (see Figure $3 \mathrm{f}$ ). The values of $T_{0}$ and $N\left(E_{\mathrm{F}}\right)$ below and above a temperature range of -120 to $-128^{\circ} \mathrm{C}$ were used to calculate the hopping distance $R$ and the average hopping energy $W$ as a function of $T$ (see Figure $3 \mathrm{~h}$ ). Large gaps in $R$ and $W$ appeared at the temperature of the crossover point in the $\ln \sigma T^{1 / 2}$ versus $T^{-1 / 4}$ curve. The small difference between the slopes of the line in Figure $3 \mathrm{f}$ and the large gap in Figure $3 \mathrm{~g}$ is because the $T^{-1 / 4}$ plot is less sensitive than the $1 / T$ plot.

The VRH theory is considered to be inappropriate for composite systems of semi-conductive fillers embedded in insulating polymers. Mott's original VRH theory ${ }^{13}$ was developed to evaluate the conductive mechanism in noncrystalline media, doped semiconductors and amorphous semiconductors.

Note that the $\ln \sigma$ versus $1 / T$ plot in Figure $3 \mathrm{~d}$ could be considered to be mildly curved rather than as two intersecting straight lines. Consequently, the second mechanism of fluctuation-induced tunneling was applied using a nonparabolic potential barrier in the form of an image-force corrected rectangular barrier. The nonparabolic barrier has been used as a rigorous potential barrier in general systems such as metal-insulator-metal and metal-semiconductormetal systems; $;^{18,23}$ however, the parabolic barrier has been applied to polymer-filler composite systems ${ }^{2-5,7,18}$ mainly because of the simple form of Equation (1).

Thermal fluctuation tunneling conduction in disordered systems For a thermal fluctuating field $\delta_{\mathrm{T}}$ that is much stronger than the applied field $\delta_{\mathrm{A}}$, a partial conductivity can be defined as

$$
\Sigma\left(\delta_{\mathrm{T}}\right)=\lim _{\delta_{\mathrm{A}} \rightarrow 0} \frac{\Delta j}{\delta_{\mathrm{A}}}=\frac{1}{2} \lim _{\delta_{\mathrm{A}} \rightarrow 0} \frac{j\left(\delta_{\mathrm{T}}+\delta_{\mathrm{A}}\right)-j\left(\delta_{\mathrm{T}}-\delta_{\mathrm{A}}\right)}{\delta_{\mathrm{A}}}=\frac{d j\left(\delta_{\mathrm{T}}\right)}{d \delta_{\mathrm{T}}}
$$

which can be used to describe the fluctuation-induced tunneling conductivity $\sigma$

$$
\sigma=\int_{0}^{\infty} P\left(\delta_{\mathrm{T}}\right) \Sigma\left(\delta_{\mathrm{T}}\right) d \delta_{\mathrm{T}}=\int_{0}^{\infty} P\left(\delta_{\mathrm{T}}\right) \frac{d j\left(\delta_{\mathrm{T}}\right)}{d \delta_{\mathrm{T}}} d \delta_{\mathrm{T}}
$$

Following Simmons, ${ }^{18}$ Sheng ${ }^{19}$ accurately expressed the shape of the potential barrier of an electric field as

$$
V(u, \bar{\delta})=V_{0}\left[1-\frac{\lambda}{u(1-u)}-\bar{\delta} u\right] V(u, \varepsilon)=V_{0}\left[1-\frac{\lambda}{u(1-u)}-\bar{\delta}_{0} \varepsilon u\right]
$$

where $u=x / D$ denotes the reduced spatial variable, $D$ denotes the insulator distance (the potential barrier width) between the two adjacent CFs, $x$ denotes the distance from the left surface of the junction, $V_{0}$ denotes the height of the rectangular potential barrier in the absence of an image-force correction, and $\bar{\delta}=\delta e D / V_{0}$ denotes the electric field expressed in the units of $\delta_{V}=V_{0} / e D$. Sheng ${ }^{19}$ showed that $\lambda=0.795 e^{2} / 4 D K V_{0}$ is an important dimensionless parameter that governs the strength of the image-force correction and the resulting barrier shape, where $e$ denotes the electron charge (which equals $1.602 \times 10^{-12} \mathrm{erg}$ ), and $K$ denotes the dielectric constant of the insulating barrier, which equals 2.3 for PE in the present calculation. The zero of the potential in Equation (11) is defined by the Fermi level of the conducting region. Sheng ${ }^{19}$ showed that the potential $V(u, \bar{\delta})$ is a peaked function of $u$, with a maximum $V_{\mathrm{m}}\left(u^{*}, \bar{\delta}\right)$, where $u^{*}$ satisfies the condition $(\partial V / \partial u)=0$. Defining $\bar{\delta}=\bar{\delta}_{0}$ at $V_{\mathrm{m}}=0$, $\bar{\delta}_{0} / \lambda$ has a physical meaning for $\lambda \leqslant 0.25$. Increasing $\lambda$ drastically increases $\bar{\delta}_{0}$, which is determined by $\lambda$. At $\lambda=0.2$, the resistivity increases drastically with the temperature from -273 to $-250{ }^{\circ} \mathrm{C}$ and levels off upon further increases in the temperature. ${ }^{19}$ This information can be used to easily determine the values of $D$ and $A$ in a computer simulation. In the present work, this information was used to obtain the best fit between the experimental and calculated results for UHMWPE-CF composite systems. Sheng ${ }^{19}$ defined a new dimensionless field parameter $\varepsilon=\bar{\delta} / \bar{\delta}_{0}$ to characterize a field such that $\varepsilon=1$ delineates a point below which $V_{\mathrm{m}}>0$ and above which $V_{\mathrm{m}}<0$. The tunneling characteristics for $\varepsilon \leqslant 1$ are drastically different from those for $\varepsilon>1$.

Sheng ${ }^{19}$ used the approximations for barrier transmission from the Wentzel-Kramers-Brillouin (WKB) approximation and the FermiDirac function to obtain an explicit expression for the tunneling current density $j(\varepsilon)$. The resulting expression for $j(\varepsilon)$ is $j_{0}(\varepsilon) \exp [-2 \chi D \xi(\varepsilon)]$ for $\varepsilon \leqslant 1$ (see Equations (A1)) and $j_{1}(\varepsilon)$ for $\varepsilon>1$ (see Equation (A2)), as shown in the Appendix. Note that $j(\varepsilon)$ is continuous at $\varepsilon=1$.

The partial conductivity $\Sigma(\varepsilon)$ can be obtained using $j(\varepsilon)$ as $\Sigma_{0}(\varepsilon) \exp [-2 \chi D \xi(\varepsilon)]$ for $\varepsilon<1$ (see Equations (A1)) and $\Sigma_{1}(\varepsilon)$ for $\varepsilon>1$ (see Equation (A2)).

Substituting Equation (10) into Equations (A6) and (A7), the conductivity $\sigma$ is given by

$$
\begin{aligned}
\sigma=\left(\frac{4 T_{1}}{\pi T}\right)^{1 / 2}\left\{\int_{0}^{1} \sum_{0}\left(\varepsilon_{\mathrm{T}}\right) \exp \left[-\frac{T_{1}}{T} \varepsilon_{\mathrm{T}}^{2}-\frac{T_{1}}{T_{0}} \varphi\left(\varepsilon_{\mathrm{T}}\right)\right] d \varepsilon_{\mathrm{T}}\right. \\
\left.+\int_{1}^{\infty} \sum_{1}\left(\varepsilon_{\mathrm{T}}\right) \exp \left(-\frac{T_{1}}{T} \varepsilon_{\mathrm{T}}^{2}\right) d \varepsilon_{\mathrm{T}}\right\},
\end{aligned}
$$

where $\varepsilon_{\mathrm{T}}=\delta_{\mathrm{T}} / \delta_{0}$ and $\varphi(\varepsilon)=\xi(\varepsilon) / \xi(0) . \varphi(\varepsilon)$ is unity at $\varepsilon=0$ and zero at $\varepsilon=1$. The new parameters $T_{1}$ and $T_{0}$ are defined as follows:

$$
\begin{aligned}
& T_{1}=\frac{D A \delta_{0}^{2}}{8 \pi k} \\
& T_{0}=\frac{T_{1}}{2 \chi D \xi(0)}
\end{aligned}
$$

where $\delta_{0}$ is given by $\bar{\delta}_{0} V_{0} / e D$ and $A$ is the surface area over which most of the tunneling occurs, as previously described.

Sheng ${ }^{19}$ reported that at small $\varepsilon_{\mathrm{T}}(<0.4)$, increasing the thermal fluctuation field caused the tunneling probability to increase faster than the thermal fluctuation probability decreased. However, at larger $\varepsilon_{\mathrm{T}}$, the reverse behavior occurred. Thus, the sum of the two factors $-\left[\left(T_{1} / T\right) \varepsilon^{2}+\left(T_{1} / T_{0}\right) \phi(\varepsilon)\right]$ in Equation (12) exhibited a maximum at the value $\varepsilon_{\mathrm{T}}=\varepsilon^{*}$. Numerical calculations clearly indicated a sharp maximum in the integrand of the first integral in Equation (12). In this case, the temperature dependence of $\sigma$ may have been dominated by the variation in $\varepsilon^{*}$. 
Using $\varepsilon^{*}$ and $\varphi\left(\varepsilon^{*}\right)$ from the discussion above, Equation (12) can be rewritten to produce an approximate expression for $\sigma$ as follows:

$$
\sigma=\sigma_{0} \exp \left[-\frac{T_{1}}{T} \varepsilon^{* 2}-\frac{T_{1}}{T_{0}} \varphi\left(\varepsilon^{*}\right)\right]
$$

where $\sigma_{0}$ is defined as

$$
\begin{aligned}
& \sigma_{0}=\left(\frac{4 T_{1}}{\pi T}\right)^{1 / 2} \exp \left(\frac{T_{1}}{T} \varepsilon^{* 2}+\frac{T_{1}}{T_{0}} \varphi\left(\varepsilon^{*}\right)\right)\left\{\int _ { 0 } ^ { 1 } d \varepsilon _ { \mathrm { T } } \sum _ { 0 } ( \varepsilon _ { \mathrm { T } } ) \operatorname { e x p } \left[-\frac{T_{1}}{T} \varepsilon_{\mathrm{T}}^{2}\right.\right. \\
&\left.\left.-\frac{T_{1}}{T_{0}} \varphi\left(\varepsilon_{\mathrm{T}}\right)\right]+\int_{1}^{\infty} d \varepsilon_{\mathrm{T}} \sum_{1}\left(\varepsilon_{\mathrm{T}}\right) \exp \left(-\frac{T_{1}}{T} \varepsilon_{\mathrm{T}}^{2}\right)\right\} .
\end{aligned}
$$

Thus, Equation (15) is an approximate description of the conductivity for a rigorous potential shape given by Equation (11). However, most studies on the conductivity of polymer-filler composites have used the parabolic barrier ${ }^{2-5,7,20,21}$ to avoid complicated formulations such as Equations (12-16). That is, the following equation by Sheng ${ }^{19}$ has generally been used, assuming a parabolic potential:

$$
V_{p}(u, \varepsilon)=V_{0}\left[u(1-u)-\varepsilon \bar{\delta}_{0} u\right] .
$$

Using Equation (17) to calculate the conductivity yields the approximation given by Equation (1).

In the theoretical calculations, Equations (A11) and (A12) were integrated from 0 to 1 using $u(=x / D)$ in intervals of $1 / 100000$. The numerical calculation of $d \eta_{1}(\varepsilon) / d \varepsilon$ in Equation (A9) to obtain the partial conductivity $\Sigma(\varepsilon)$ for $\varepsilon>1$ was rather complex. The calculation was performed using the Origin Pro 8 software and Fortran 77.

The partial conductivity $\Sigma(\varepsilon)$ for $\varepsilon>1$ was found to be negligibly smaller than $\Sigma(\varepsilon)$ for $\varepsilon \leqslant 1$; therefore, the numerical calculation discussed below was performed only for $\varepsilon \leqslant 1$.

Figure $4 \mathrm{a}$ shows a plot of the conductivity $(\sigma)$ versus the temperature $(T)$, as calculated by Equation (12), and Figure $4 \mathrm{~b}$ shows the corresponding logarithmic plots of $\sigma$ versus $1 / T$ for a UHMWPECF composite with a $99-$ vol\% CF content. The experimental results (shown as black open circles) at $0.4 \mathrm{~V}$ from Figure $3 \mathrm{~b}$ are replotted in this figure. The theoretical calculation (shown by a red solid curve) was performed at an applied voltage of $0.4 \mathrm{~V}$ to avoid the effect of the applied field.

The parameters $\lambda\left(=0.795 e^{2} / 4 D K V_{0}\right), D$ and $A$ were determined to perform the numerical calculation. The aforementioned increase in the conductivity with the temperature was used to postulate a $\lambda$ value between 0.05 and 0.1 for the composites with 25 and $30 \mathrm{vol} \% \mathrm{CF}$ content and between 0.1 and 0.2 for the composite with a $99-\mathrm{vol} \% \mathrm{CF}$ content. The $D$ and $A$ values were determined by a computer simulation. The simulation to fit the theoretical (red solid curve) results to the experimental data (black open circles) showed that $D$ was the most sensitive and important parameter for obtaining the best fit, whereas $\lambda$ and $A$ were not sensitive compared with $D$. Figure $5 \mathrm{a}$ shows that the best fits were obtained using a constant $D$-value for temperatures $<-80^{\circ} \mathrm{C}$, whereas a temperature-dependent $D$ had to be used for temperatures greater than $-80^{\circ} \mathrm{C}$. That is, using $D$ values other than those in Figure $5 \mathrm{a}$ did not produce a good fit despite adjusting the $\lambda$ and $A$ values. The best fit was obtained for $D=0.500$ $\mathrm{nm}$ in the temperature range from -146 to $-80^{\circ} \mathrm{C}$, for $\lambda=0.1201$ and an $A$ value of $3.00 \mathrm{~nm}^{2}$. Figure 4 shows that the agreement between theory and experiment was improved for temperatures above $-80^{\circ} \mathrm{C}$ by slightly changing $D$ at $\lambda=0.1201$ and $A=3.00 \mathrm{~nm}^{2}$ as in Figure 5a. A good fit could not be obtained for $D \neq 0.500 \mathrm{~nm}$ for any values of $\lambda$ and $A$ over the temperature range from -146 to $-80^{\circ} \mathrm{C}$.
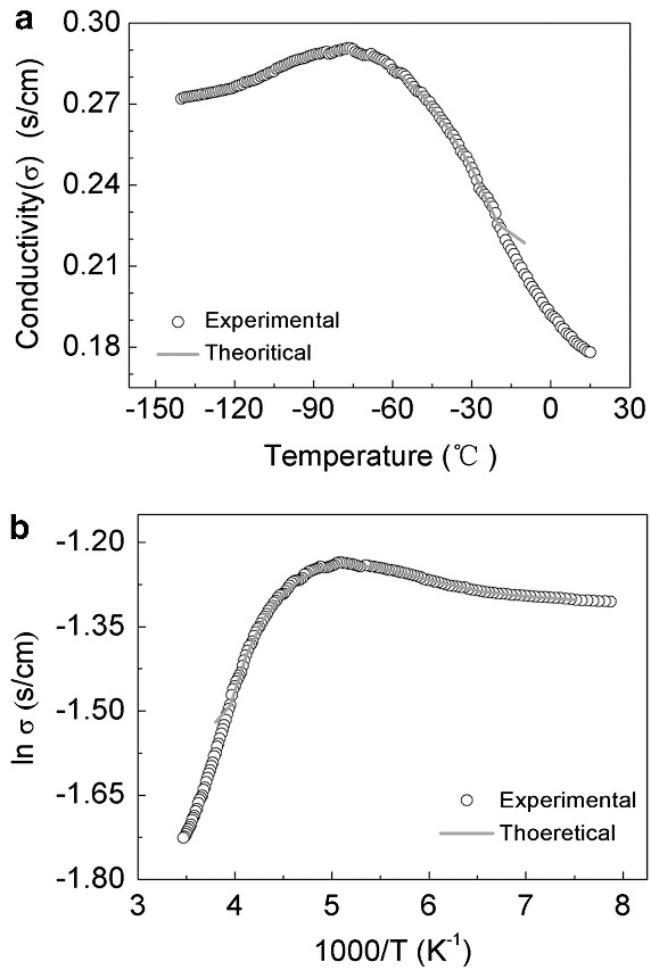

Figure 4 Experimental measurements (black open circles) and theoretical (red solid line) results using Equations (12) or (15) for a composite with a 99-vol\% carbon fiber (CF) content: (a) conductivity versus temperature and (b) In $\sigma$ versus $1000 / T$. A full color version of this figure is available at Polymer Journal online.

The barrier width $D$ was considered to be the average contact distance between adjacent CFs during the preparation of the 99 vol\% CF film, which was molded in two compression steps, that is, compression under $2 \mathrm{MPa}$ at $180^{\circ} \mathrm{C}$ for $10 \mathrm{~min}$, followed by compression under $5 \mathrm{MPa}$ for $20 \mathrm{~min}$ after annealing from $180^{\circ} \mathrm{C}$ to room temperature. Figure $5 \mathrm{~b}$ shows the potential barrier calculated by Equation (11) for $D=0.500 \mathrm{~nm}, \lambda=0.1201$, and $A=3.00 \mathrm{~nm}^{2}$. A nonparabolic barrier was used to obtain Equation (11); therefore, the potential barrier $V(u, \varepsilon)$ was negative near $u=0$ and unity. Increasing $\varepsilon$ caused the barrier to become asymmetrical and shifted the maximum peak $\left(V_{\mathrm{m}}\right)$ to lower $u$ values, also decreasing $V_{\mathrm{m}}$

Figure $4 \mathrm{a}$ shows that the conductivity decreased above $-80^{\circ} \mathrm{C}$. This decreased conductivity was most likely caused by the decreased adhesion power of $1 \mathrm{vol} \%$ UHMWPE to connect adjacent CFs above $-80^{\circ} \mathrm{C}$ and the increased thermal expansion of the air voids between the adjacent CFs. This behavior implied that $D$ should increase with temperature, as was observed in Figure 5a. Figure 4 shows that the conductivity of the CFs carbonized above $2000^{\circ} \mathrm{C}$ was considerably lower than the in-plane conductivity of a single wall carbon nanotube. ${ }^{41}$

Figures $6 \mathrm{a}$ and $\mathrm{b}$ show the temperature-dependent conductivity of the UHMWPE-CF composites with a $30-\mathrm{vol} \%$ CF content at the voltages indicated for two specimen types with crystallinities of $51.4 \%$ and $34.6 \%$, respectively, as measured by differential scanning calorimetry. The conductivity increased gradually with the temperature and attained a maximum between -15 and $0{ }^{\circ} \mathrm{C}$. At the voltages indicated, the conductivity of the composite with $51.4 \%$ crystallinity was higher than that of the composite with $34.6 \%$ crystallinity, indicating that the crystal region presented a slightly lower potential 
barrier to electron transport than the amorphous region. Thus, the crystal region in the UHMWPE medium transmitted electrons more easily than the amorphous region. ${ }^{42}$ The gradual increase in the
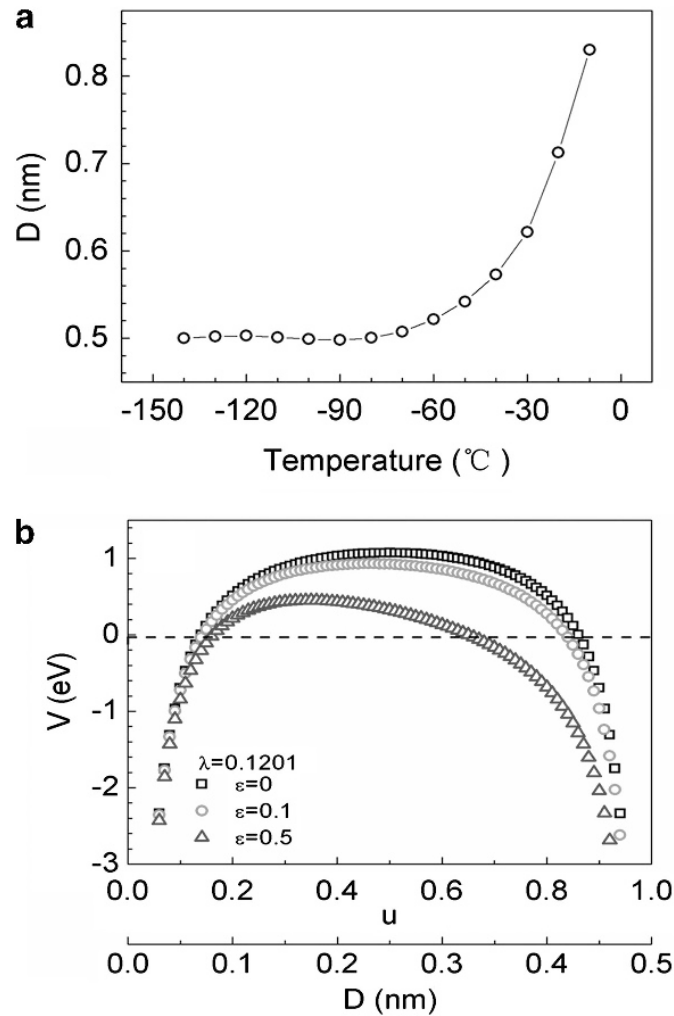

Figure 5 Calculated parameters for a composite with a 99-vol\% carbon fiber (CF) content: (a) $D$ (barrier distance width) versus temperature and (b) corresponding $V$ versus $D$ (and $u$ ), calculated using Equation (11) at the indicated $\varepsilon$ values and $D=0.500 \mathrm{~nm}$. A full color version of this figure is available at Polymer Journal online.

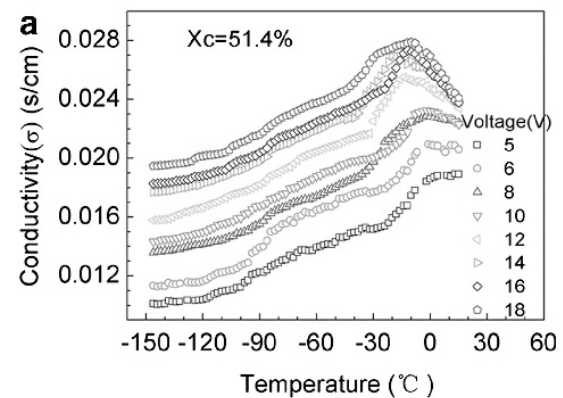

C

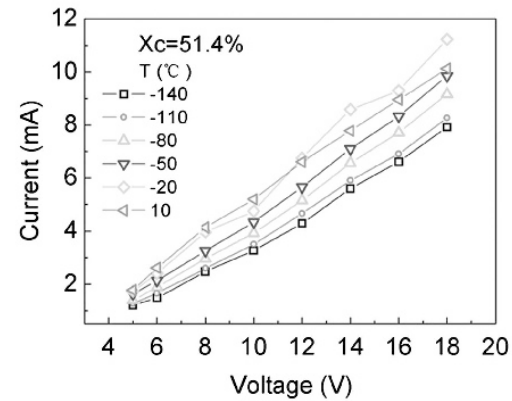

conductivity up to -15 to $0{ }^{\circ} \mathrm{C}$ may be attributed to the active electron transmission that was accelerated by the tunneling effect. However, the conductivity decreased as the temperature increased above -15 to $0{ }^{\circ} \mathrm{C}$, indicating thermal expansion and/or the active molecular mobility of the UHMWPE amorphous chains from macroBrownian motion. For the specimens with $51.4 \%$ crystallinity in Figure $6 \mathrm{c}$ and $34.6 \%$ crystallinity in Figure $6 \mathrm{~d}$, the relationship between the current and the voltage at the indicated temperature was nonlinear and resembled an exponential function, confirming the tunneling effect.

The plots for the specimen with $51.4 \%$ crystallinity show considerable scatter relative to the plot for the specimen with $34.6 \%$ crystallinity. The scatter was most likely caused by the considerable mechanical relaxation (dispersion) peak for the specimen with $51.4 \%$ crystallinity, which shall be discussed later in terms of the sensitive temperature dependence of the loss modulus in Figure 11.

Figures $7 \mathrm{a}$ and $\mathrm{b}$ show the temperature-dependent conductivity $(\sigma)$ calculated by Equation (12) for the $30 \mathrm{vol} \%$ CF composite with $51.4 \%$ and $34.6 \%$ crystallinity, respectively; Figures $7 \mathrm{c}$ and $\mathrm{d}$ show the corresponding natural logarithmic plots of $\sigma$ versus $1 / T$.

The best fit between the experimental (black open circles) and theoretical (red solid line) results by computer simulation was obtained using a $D$-value of $1.00 \mathrm{~nm}$ for both specimens and $\lambda$ values of 0.080 and 0.070 for the specimens with $51.4 \%$ and $34.6 \%$ crystallinity, respectively. Figures $7 \mathrm{a}$ and $\mathrm{b}$ show the best fits for the specimens with $51.4 \%$ and $34.6 \%$ crystallinity, respectively. The best fit for the specimen with $51.4 \%$ crystallinity was obtained for $D=1.00 \mathrm{~nm}, \lambda=0.080$ and $A=1.64$ to $1.68 \mathrm{~nm}^{2}$, and the best fit for the specimen with $34.6 \%$ crystallinity was obtained for $D=1.00 \mathrm{~nm}$, $\lambda=0.070$ and $A=1.35$ to $1.40 \mathrm{~nm}^{2}$ up to $0{ }^{\circ} \mathrm{C}$, as shown in Figure 8 a.

Figure 8 a shows that making a small adjustment in $A$ produced the best fit in the temperature range considered. Note that a $D$-value of $1.00 \mathrm{~nm}$ was required to obtain a good fit for the film with a $30-\mathrm{vol} \%$ $\mathrm{CF}$ content; the theoretical results deviated considerably from the experimental data for $D$ values above or below $1.00 \mathrm{~nm}$, despite adjustments in $\lambda$ and $A$. The parameter sets in Figure $8 \mathrm{a}$ also ensured
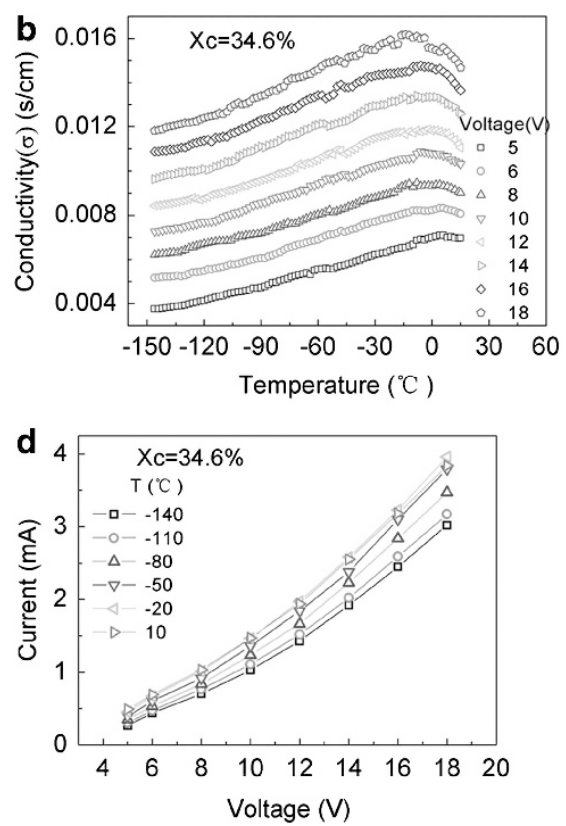

Figure 6 Conductivity versus temperature $\left(T\right.$ ) for composites with a 30 -vol\% carbon fiber (CF) content for (a) $X_{C}=51.4 \%$ and (b) $X \mathrm{C}=34.6 \%$; corresponding current versus voltage for (c) $X_{c}=51.4 \%$ and (d) $X_{c}=34.6 \%$. A full color version of this figure is available at Polymer Journal online. 

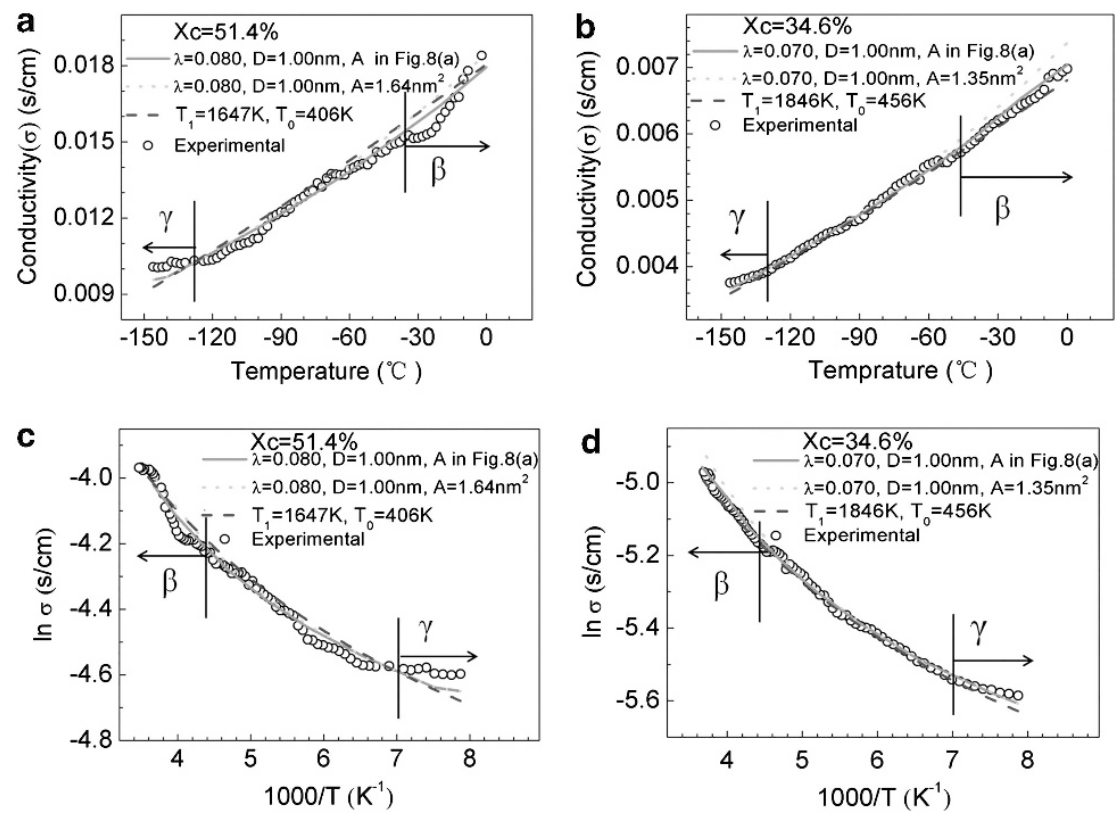

Figure 7 Experimental and theoretical results using Equations (12) or (15) using the indicated parameters for composites with a 30-vol\% carbon fiber (CF) content for (a) $X_{\mathrm{c}}=51.4 \%$ and (b) $X \mathrm{c}=34.6 \%$; corresponding $I n \sigma$ versus $1000 / T$ plots for (c) $X_{\mathrm{C}}=51.4 \%$ and (d) $X \mathrm{c}=34.6 \%$, in which the red solid line was calculated by adjusting $A$ in Figure 8 a, the green dotted line was calculated using a fixed $A$ value, and the blue dashed line was calculated using Equation (1). A full color version of this figure is available at Polymer Journal online.

good agreement between the theoretical and experimental results for the logarithmic plots of $\sigma$ versus $1 / T$ in Figures $7 \mathrm{a}$ and $\mathrm{b}$.

A reasonably good fit was obtained using fixed values of $D, \lambda$ and A. The green dotted lines in Figure 7 , which were drawn without adjusting $A$, agreed reasonably well with the experimental results using $D=1.00 \mathrm{~nm}, \lambda=0.080$ and $A=1.64 \mathrm{~nm}^{2}$ for the specimen with $51.4 \%$ crystallinity and using $D=1.00 \mathrm{~nm}, \lambda=0.070$ and $A=$ $1.35 \mathrm{~nm}^{2}$ for the specimen with $34.6 \%$ crystallinity. The blue dashed lines, which were obtained using Equation (1), shall be discussed later.

Figures $8 \mathrm{~b}$ and $\mathrm{c}$ show the shape of the potential barrier at $\varepsilon=0$, 0.1 and 0.5 . Note that $V_{\mathrm{m}}$ at $\lambda=0.080$ in Figure $8 \mathrm{~b}$ was slightly higher than $V_{\mathrm{m}}$ at $\lambda=0.070$ in Figure $8 \mathrm{c}$. Increasing $\varepsilon$ caused the potential barrier $V(E, \varepsilon)$ to become asymmetrical by shifting $V_{\mathrm{m}}$ to lower $u$ values, thereby decreasing $V_{\mathrm{m}}$. In comparison, the barrier height in Figure $5 \mathrm{~b}$ increased slightly as $D$ increased.

Figure 7 shows that the theoretical results calculated using a weakly temperature-dependent $A$ value deviated slightly from the experimental results at temperatures less than $-130{ }^{\circ} \mathrm{C}$ and greater than $-24{ }^{\circ} \mathrm{C}$ for the composite with $51.4 \%$ crystallinity and at temperatures less than $-130^{\circ} \mathrm{C}$ and greater than $-45^{\circ} \mathrm{C}$ for the composite with $34.6 \%$ crystallinity. The deviations at low and high temperatures were attributed to the $\gamma$ and $\beta$ relaxations shown in Figure 7. The origin of these relaxations was investigated by probing structural changes in the PE matrix by positron annihilation and complex dynamic modulus measurements, which shall be discussed later.

Before presenting the relationship between the conductivity and the molecular mobility of the PE matrix, we present results for the temperature-dependent conductivity of the composite with a $25-\mathrm{vol} \%$ CF content to justify the use of a nonparabolic potential barrier in Equation (12).

Figures $9 \mathrm{a}$ and $\mathrm{b}$ show the temperature-dependent conductivity $(\sigma)$ and logarithmic plots of $\sigma$ versus $1 / T$ and the best fits between the theoretical (red solid line) and experimental (black open circles) results from computer simulation. The best fits were obtained at $\lambda=0.055, D=1.30 \mathrm{~nm}$, and $A=1.25$ to $1.28 \mathrm{~nm}^{2}$. Figure $10 \mathrm{a}$ shows the slight adjustment in $A$.

Figure 10a shows that a small adjustment in $A$ produced the best fit over the temperature range considered. A $D$-value of $1.30 \mathrm{~nm}$ was used, similar to the composites with a $30-\mathrm{vol} \%$ CF content shown in Figure 8a; however, a considerable deviation between the theoretical and experimental results was observed for $D$ values above or below $1.30 \mathrm{~nm}$, despite adjustments in $\lambda$ and $A$.

The computer simulations for the parameter fitting were straightforward because $\lambda$ was estimated from the slope of the logarithmic plots of $\sigma$ versus $1 / T$. As previously discussed, $\lambda$ must be below 0.25 from theoretical considerations. For insulating polymers, $\lambda$ decreases as the matrix content increases, whereas $\lambda$ is 0.2 for materials similar to super-conductors, in which the resistivity increases over temperatures ranging from -273 to $-250{ }^{\circ} \mathrm{C}$ and levels off above $-250{ }^{\circ} \mathrm{C} .{ }^{19}$ Therefore, the computer simulations to fit the $D$ and $A$ parameters were straightforward.

The green dotted lines in Figure 9, which were drawn without adjusting $A$, agreed reasonably well with the experimental results for $\lambda=0.055, D=1.30 \mathrm{~nm}$ and $A=1.25 \mathrm{~nm}^{2}$. The blue dashed lines obtained using Equation (1) shall be discussed later along with the blue lines in Figure 7.

Figure $10 \mathrm{~b}$ shows the shape of the potential barrier at $\varepsilon=0,0.1$, and 0.5 . The value of $V_{\mathrm{m}}$ at $\lambda=0.055$ was slightly higher than at $\lambda=0.080$ and 0.070 in Figures $8 \mathrm{~b}$ and $\mathrm{c}$. The potential barrier $V(E, \varepsilon)$ became asymmetrical because $V_{\mathrm{m}}$ shifted to lower $u$ values, thereby decreasing $V_{\mathrm{m}}$. Comparing Figure $5 \mathrm{~b}$ with Figures $8 \mathrm{~b}$ and $\mathrm{c}$ shows that $V_{\mathrm{m}}$ was higher at $\varepsilon=0$ but decreased at $\varepsilon=0.5$ as $D$ increased.

The good fits showed that the percolation threshold could clearly be identified with the lowest CF content, which showed that there were sufficient junctions with barrier widths $(1.30 \mathrm{~nm})$ between adjacent CFs associated with electron transmission.

The potential barrier width $D$ was 1.30 and $1.00 \mathrm{~nm}$ for the composites with a 25 and $30 \mathrm{vol} \%$ CF content, respectively. This 

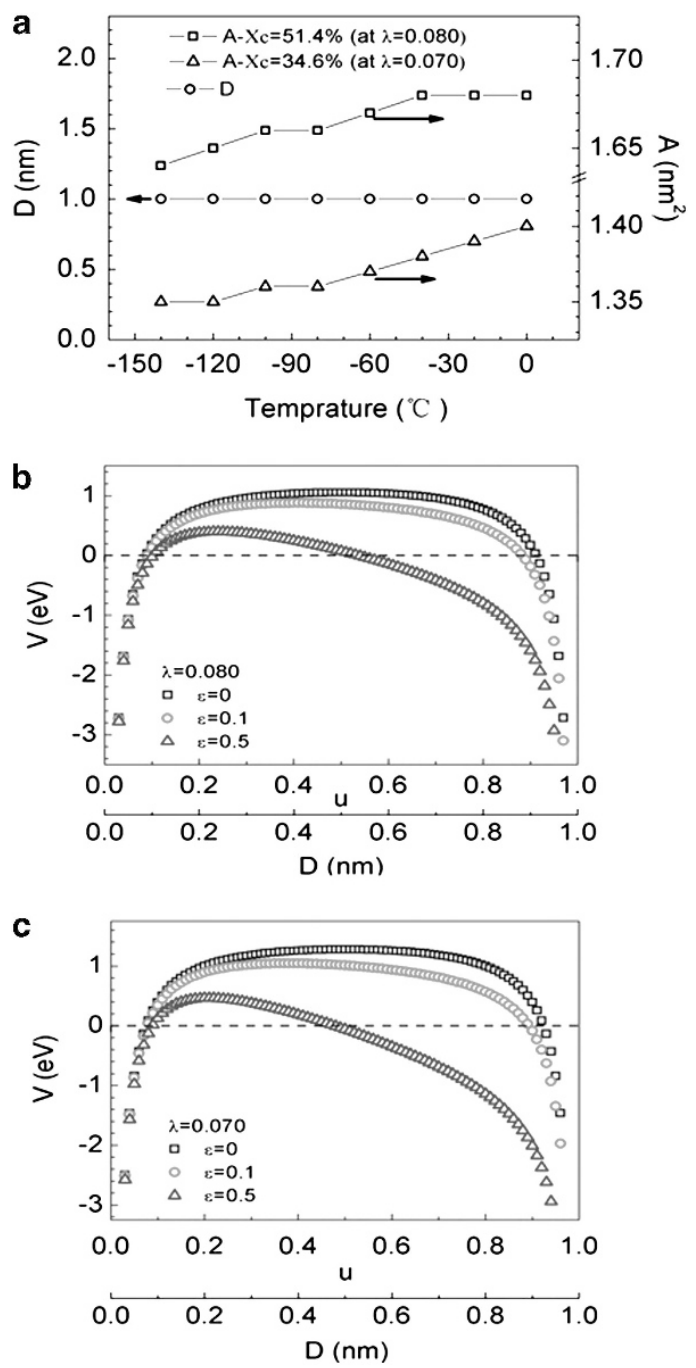

Figure 8 Calculated parameters for a composite with a 99-vol\% carbon fiber (CF) content: (a) $D$ and $A$ values versus temperature for $\lambda=0.080$ at $X \mathrm{C}=51.4 \%$ and for $\lambda=0.070$ at $X \mathrm{c}=34.6 \%$; (b) and (c), corresponding $V$ versus $D$ (and $u$ ) plots calculated using Equation (11) at the indicated $\varepsilon$ values for composites with a 30-vol\% CF content. A full color version of this figure is available at Polymer Journal online.

difference is typical because the distance between the neighboring CFs at a $25-\mathrm{vol} \% \mathrm{CF}$ content was greater than at a $30-\mathrm{vol} \% \mathrm{CF}$ content.

Positron annihilation is a useful tool for probing the free volume in a polymer medium. The histogram in Figure 11a shows that the free volume estimated by the long-lived life-time $\left(\tau_{3}\right)$ in a UHMWPE medium increased with temperature; the estimation method and the maximum entropy for lifetime analysis of the results have been described elsewhere. ${ }^{43}$ The horizontal scale corresponds to the radius of free volume from Tao's equation. ${ }^{44}$ The sharpness of the intensity distribution was indicative of a narrow distribution of holes of a relatively uniform size. The figure shows that the peak shifted to higher $\tau_{3}$ values with temperature, indicating a larger free volume. The histograms show that the average change in the free volume diameter in the medium ranged from $\sim 0.5$ to $0.68 \mathrm{~nm}$ over the temperature range considered $\left(-150\right.$ to $\left.25^{\circ} \mathrm{C}\right)$. Thus, the increase in conductivity with temperature was attributed to the acceleration of the thermal fluctuation field in surmounting the thermal expansion of the barrier width, which increased the free volume size.
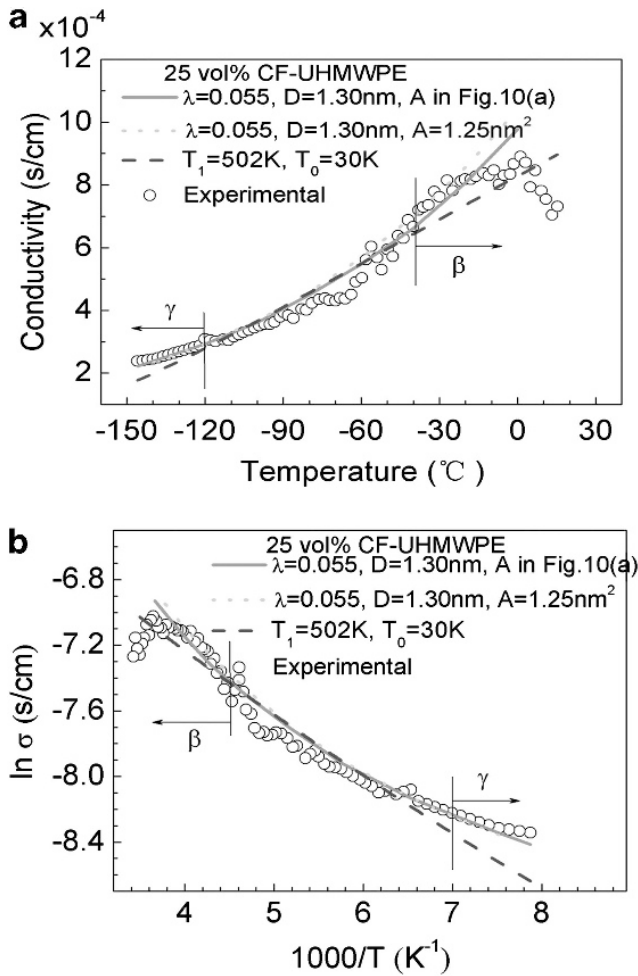

Figure 9 (a) Experimental and theoretical results using Equation (12) using the indicated parameters for composites with a $25-\mathrm{vol} \%$ carbon fiber (CF) content; (b) corresponding plot of In $\sigma$ versus $1000 / T$, in which the red solid line was calculated by adjusting $A$ in Figure 10a, the green dotted line was calculated using a fixed value of $A$, and the blue dashed line was calculated using Equation (1). A full color version of this figure is available at Polymer Journal online.

Figure $11 \mathrm{~b}$ shows the variation in the o-Ps intensity $\left(I_{3}\right)$ with temperature. Figures $11 \mathrm{c}$ and $\mathrm{d}$ show the temperature-dependent storage modulus $E^{\prime}$ and loss modulus $E^{\prime \prime}$, respectively, for the composite with a $30-$ vol\% CF content. Similar profiles were obtained for the composite with a $25-\mathrm{vol} \%$ CF content, which is not shown here for brevity. As previously reported, $E^{\prime}$ decreased drastically at temperatures near the $\beta$ and $\gamma$ relaxation peaks. ${ }^{45}$ The $\beta$ relaxation, which appeared at approximately $-10^{\circ} \mathrm{C}$, was associated with the large (macro-Brownian) motion of the amorphous UHMWPE chains and an increase in the amorphous volume. The $\beta$ relaxation peak corresponded to the minimum $o$-Ps intensity $\left(I_{3}\right)$ because most of the shallow potentials may have been smeared out by the local motion, causing the trapped electrons to also disappear. The large motion caused the potential barrier width to increase drastically and the conductivity to decrease above $-10^{\circ} \mathrm{C}$, as shown in Figure 6 . However, the $\gamma$ relaxation involves a rapid trans-gauche transition of the central C-C bonds of a short amorphous segment (for example, three to four $\mathrm{CH}_{2}$ groups). ${ }^{45}$ Therefore, the $\gamma$ relaxation peak of the composite with $34.6 \%$ crystallinity was higher than that of the composite with $51.4 \%$ crystallinity. The small motion associated with the $\gamma$ relaxation did not greatly affect the potential barrier width, resulting in the theoretical conductivity results deviating from the experimental data. The theoretical results agreed poorly with the experimental results, despite attempts to adjust the parameters in the temperature range around the $\beta$ and $\gamma$ relaxation peaks, as shown in Figure 7. Fluctuation-induced tunneling conduction in disordered 

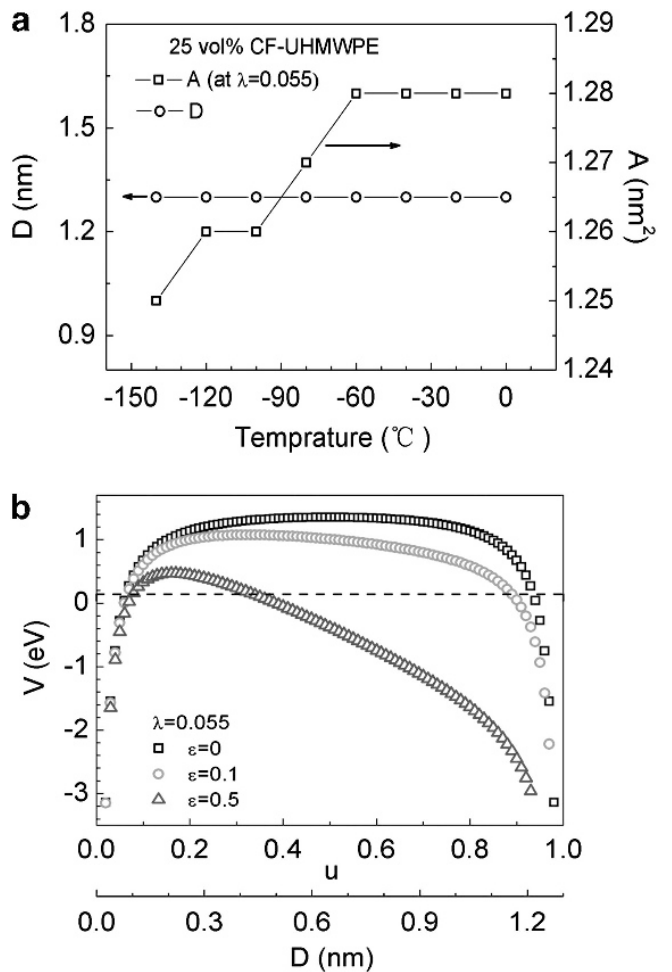

Figure 10 (a) Calculated $D$ and $A$ values versus temperature at $\lambda=0.055$ for a composite with a 25-vol\% carbon fiber (CF) content and (b) corresponding $V$ versus $D$ (and $u$ ) plot calculated using Equation (11) at the indicated $\varepsilon$ values for a composite with a $25-$ vol\% CF content. A full color version of this figure is available at Polymer Journal online. materials was originally developed for electron transmission because of thermal fluctuations; therefore, the drastic change in the molecular mobility over specific temperature ranges, such as the $\beta$ and $\gamma$ relaxations in PE, do not conform to the original framework of the theory. The increased scatter in the conductivity values for the specimen with the $51.4 \%$ cystallinity relative to the specimen with $34.6 \%$ crystallinity in Figure 6 most likely originated from the sharp dispersion profile of the $\beta$ relaxation over the temperature range from -40 to $20^{\circ} \mathrm{C}$.

Note that the conductivity values in Figures 4 to 10 at 30 and $25 \mathrm{vol} \% \mathrm{CF}$ content increased up to $-10{ }^{\circ} \mathrm{C}$, whereas the conductivity values for the composites with $99 \mathrm{vol} \% \mathrm{CFs}$ decreased above $-80^{\circ} \mathrm{C}$. This large difference was because there were fewer air voids between adjacent CFs at 30 vol\% CF than at 99 vol\% CF. Thus, adjacent CFs were connected strongly to each other by the adhesion power of the 70 vol\% UHMWPE, whereas the adhesion power between adjacent CFs for 1 vol\% UHMWPE with 99 vol\% CF content was less effective above $-80^{\circ} \mathrm{C}$, and the thermal expansion of air voids between adjacent CFs was accelerated.

Approximations are required to obtain the barrier transmission factor $\operatorname{TF}(E, \varepsilon)$ from the rigorous potential barrier in Equation (11); the resulting equation is simpler than the well-known WKB approximation. However, Sheng's assumption ${ }^{19}$ can be justified with very little error. This assumption is given by

$$
T F(E, \delta) \cong \begin{array}{cc}
\exp [-F(E, \varepsilon)] & E \leqslant V m \\
1 & E>V m
\end{array}
$$

where $F(E, \varepsilon)$ is given by

$$
F(E, \varepsilon) \cong 2 \chi D \xi(\varepsilon)-\frac{2 \chi D}{V_{0}} \eta_{0}(\varepsilon) E
$$
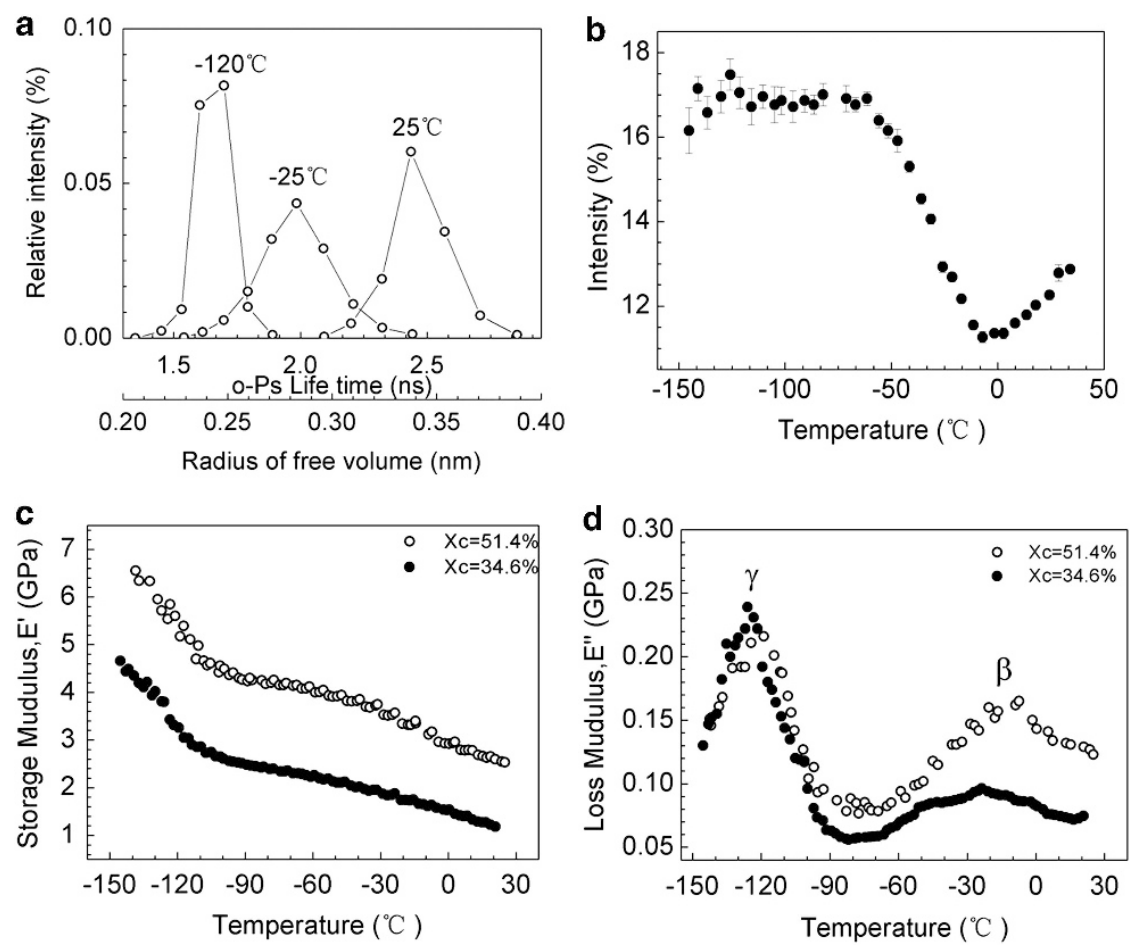

Figure 11 Physical properties of ultra-high molecular weight polyethylene (UHMWPE): (a) histogram of the life time $\left(\tau_{3}\right)$ distribution and radius of free volume; ${ }^{43}$ (b) o-PS intensity $\left(I_{3}\right)$ as a function of temperature; ${ }^{43}$ (c) temperature-dependent $E$; and (d) temperature-dependent $E^{\prime \prime}$. 

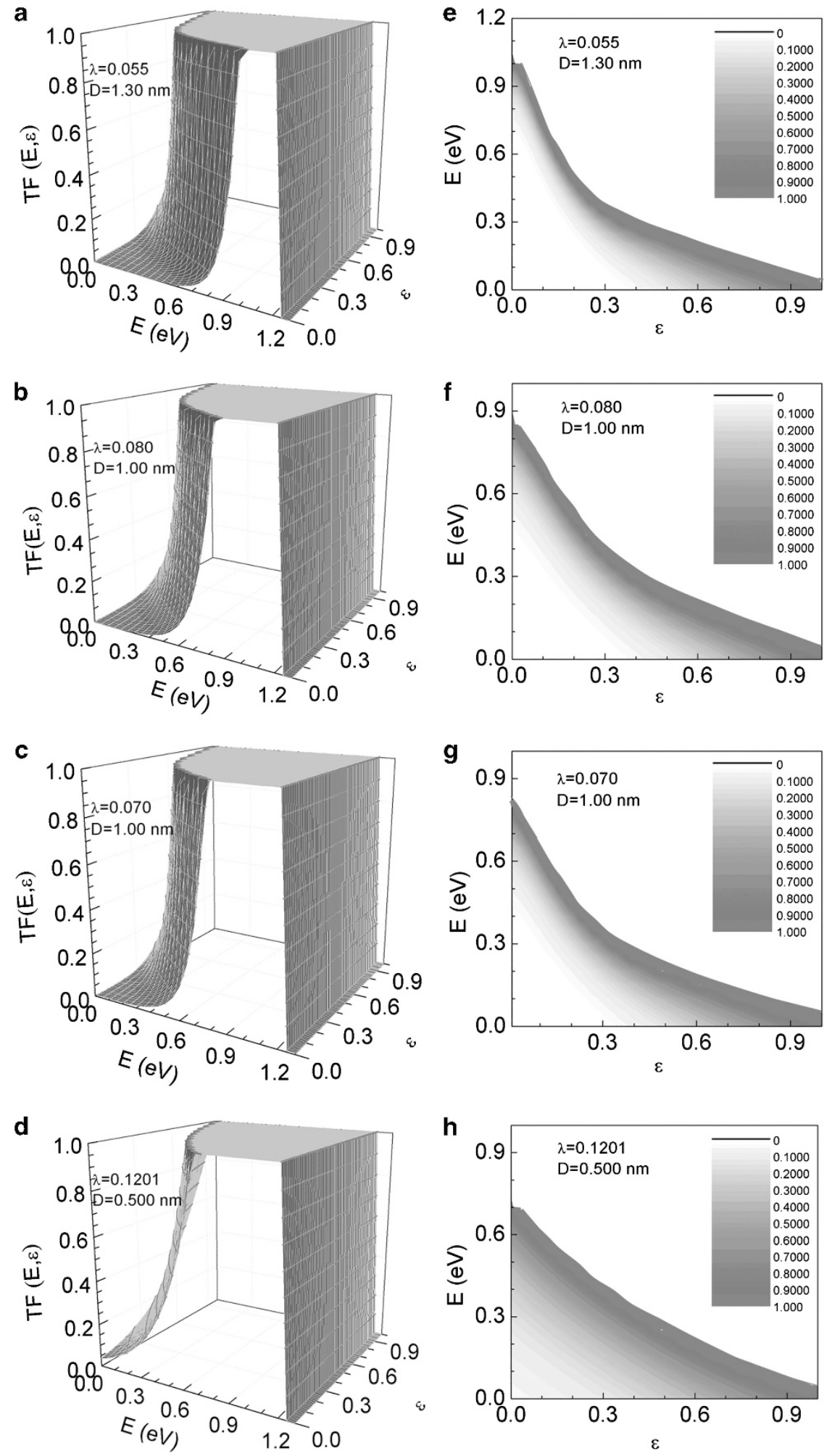

Figure 12 Solid figures of $\operatorname{TF}(E, \varepsilon)$ versus $E$ and $\varepsilon:$ (a) $\lambda=0.055$ for a composite with a 25 -vol\% carbon fiber (CF) content $(X \mathrm{C}=52.3 \%$ ); (b) $\lambda=0.080$ for a composite with a 30-vol\% CF content $\left(X_{C}=51.4 \%\right)$; (c) $\lambda=0.070$ for a composite with a $30-$ vol\% CF content $\left(X_{C}=34.6 \%\right)$; (d) $\lambda=0.1201$ for a composite with a 99-vol\% CF content; and contour maps (e), (f), (g) and (h) corresponding to the solid figures in (a), (b), (c) and (d), respectively. A full color version of this figure is available at Polymer Journal online.

Figure 12 shows $T F(E, \varepsilon)$ versus $E$ and $\varepsilon$. The solid figures in Figure $12 \mathrm{a}$ correspond to the composite with a $25-\mathrm{vol} \%$ CF content and $52.3 \%$ crystallinity, and Figures $12 \mathrm{~b}$ and $\mathrm{c}$ show the composites with a $30-$ vol\% CF content and $51.4 \%$ and $34.6 \%$ crystallinity, respectively. The solid figure in Figure $12 \mathrm{~d}$ corresponds to the molded film with a $99-$ vol\% CF content. Figures $12 \mathrm{e}-\mathrm{h}$ show the 
corresponding $T F(E, \varepsilon)$ contour maps on the $E-\varepsilon$ plane. As previously discussed, the barrier width $D$ was 1.30 and $1.00 \mathrm{~nm}$ at $25 \mathrm{vol} \%$ and 30 vol\% CF content, respectively. The $D$-value was $0.500 \mathrm{~nm}$ at 99 vol\% CF content. The $E$ value at $\operatorname{TF}(E, \varepsilon)=1$ decreased with decreasing $D$ because of smooth electron transmission. That is, when $\varepsilon=0$, $\operatorname{TF}(E, \varepsilon)=1$ was realized at $E \approx 0.95 \mathrm{eV}$ for $D=1.30 \mathrm{~nm}$ and $\lambda=0.055$, at $E \approx 0.90 \mathrm{eV}$ for $D=1.00 \mathrm{~nm}$ and $\lambda=0.080$, at $E \approx 0.80 \mathrm{eV}$ for $D=1.00 \mathrm{~nm}$ and $\lambda=0.070$, and $\mathrm{E} \approx 0.75 \mathrm{eV}$ for $D=0.500 \mathrm{~nm}$ and $\lambda=0.1201$. The electron transmission for the 99 vol\% CF molded film with a barrier width of $0.500 \mathrm{~nm}$ was significantly accelerated relative to the two types of composites with a 30-vol\% CF content and a barrier width of $1.00 \mathrm{~nm}$, confirming the conventional perspective that decreasing the barrier width accelerates the tunneling current flow. Comparing Figures $8 \mathrm{~b}$ and $\mathrm{c}$, Figure $10 \mathrm{~b}$, and Figure $5 \mathrm{~b}$ shows that the potential barrier shape $V(E, \varepsilon)$ was sensitive to $\varepsilon$. Increasing $\varepsilon$ shifted the peak $\left(V_{\mathrm{m}}\right)$ to lower $u$ values (that is, away from $u=0.5$ ), causing the curve to become asymmetrical and decreasing $V_{\mathrm{m}}$. Thus, $T F(E, \varepsilon)$ attained unity at lower $E$ values with increasing $\varepsilon$.

Finally, the use of Equation (1), which is derived for a parabolic potential barrier, is strongly cautioned. The theoretical results calculated using Equation (1) are shown as a blue dashed line for the composites with a $30-\mathrm{vol} \%$ CF content in Figure 7 and for the composites with a 25-vol\% CF content in Figure 9; note that the fits to the experimental results (shown as a green dotted line in Figures $7 \mathrm{c}$ and d) were equally as good using $D=1.00 \mathrm{~nm}, \lambda=0.080$ and $A=1.64 \mathrm{~nm}^{2}$ at $51.4 \%$ crystallinity as for $D=1.00 \mathrm{~nm}, \lambda=0.070$ and $A=1.35 \mathrm{~nm}^{2}$ at $34.6 \%$ crystallinity. Figures $9 \mathrm{a}$ and $\mathrm{b}$ show the fits that were obtained using $D=1.30 \mathrm{~nm}, \lambda=0.055$ and $A=1.25 \mathrm{~nm}^{2}$ for the composite with a 25 -vol\% CF content.

Note that the $D$ and $A$ values calculated by using $T_{0}$ and $T_{1}$ in Equation (1) were as follows: 0.279 and $0.00206 \mathrm{~nm}^{2}$, respectively, at a 30-vol\% CF content and 51.4\% crystallinity; 0.216 and $0.0104 \mathrm{~nm}^{2}$, respectively, at $34.6 \%$ crystallinity; and 0.827 and $0.0108 \mathrm{~nm}^{2}$, respectively at a $25-$ vol\% CF content; the blue dashed line obtained using Equation (1) was in good agreement with the experimental results (black open circles). These values were patently unreasonable; however, many reports have evaluated Equation (1) without discussing the $D$ and $A$ values. For example, the $D$ values at $30 \mathrm{vol} \% \mathrm{CF}$ content were both slightly higher and slightly lower than the length $(0.2354 \mathrm{~nm})$ of the $c$-axis in a PE crystal unit, and the values of $A$ were considerably less than the area $\left(0.3648 \mathrm{~nm}^{2}\right)$ of the $a-b$ plane of a PE crystal unit. Therefore, the conductivity of the composites should be evaluated using Equations (12) or (15), which were derived using a nonparabolic potential barrier such as that in Equation (11). Equation (1) was derived from Equation (15) assuming that the integrand of the first integral in Equation (12) was strongly peaked and that the temperature dependence of $\sigma$ was dominated by the variation in $\varepsilon^{*}$, resulting in a maximum value of $-\left[\left(T_{1} / T\right) \varepsilon^{2}+\left(T_{1} / T_{0}\right) \phi(\varepsilon)\right]$ in Equation (12).

Equation (17) has sometimes been used to approximate the imageforce corrected rectangular barrier, ${ }^{18,19}$ which is given as $\varphi(\varepsilon)=$ $\xi(\varepsilon) / \xi(0)$ by assuming $\bar{\delta}_{0}=1$. Then,

$$
\varphi(\varepsilon)=\frac{\int_{0}^{1-\varepsilon}[u(1-u)-\varepsilon u]^{1 / 2} d u}{\int_{0}^{1}[u(1-u)]^{1 / 2} d u}=(1-\varepsilon)^{2} .
$$

Using the maximum value of $-\left[\left(T_{1} / T\right) \varepsilon^{2}+\left(T_{1} / T_{0}\right) \phi(\varepsilon)\right]$ at $\varepsilon_{\mathrm{T}}=$ $\varepsilon^{*}$, the function $f(\varepsilon)$ has a similar form to the blanket of Equation (15):

$$
\begin{aligned}
& f(\varepsilon)=-\frac{T_{1}}{T}(\varepsilon)^{2}-\frac{T_{1}}{T_{0}} \varphi(\varepsilon) . \\
& \text { Thus }\left.\frac{d f}{d \varepsilon}\right|_{\varepsilon^{*}}=0, \text { and } \varepsilon^{*}=T /\left(T+T_{0}\right) .
\end{aligned}
$$

Substituting these relations into Equation (15) produces Equation (1). However, assumptions were made in deriving Equation (1) from Equation (15); therefore, Equation (1) may not accurately evaluate the tunneling barrier width $D$ and the surface area $A$ over which most of the tunneling occurs.

A series of results was used to attribute the conductivity of UHMWPE-CF composites to the tunneling effect from a thermal fluctuating field; the experimental results were accurately fit by a theory using a nonparabolic potential barrier. However, a small deviation between the experimental and theoretical results was observed over temperature ranges around the $\beta$ and the $\gamma$ relaxation peaks, which was related to the macro- and micro-Brownian motion of the amorphous chains. These relaxations, which are associated with the intrinsic behavior of PE, do not lie within the framework of fluctuation-induced tunneling conduction theory. In this paper, the potential barrier width between the adjacent CFs associated with electron transmission was estimated for a typical insulating UHMWPE medium; higher $D$ and $A$ values may be obtained by choosing polymers with higher matrix conductivities. Other polymers should be investigated in the future.

\section{CONCLUSION}

UHMWPE-CF composites were prepared from solution using a gelation/crystallization method. Classical percolation theory was used to select CF contents of 25 and $30 \mathrm{vol} \%$ for the composites in the study. To elucidate the conductive mechanism in the composites, the conductivities of a long CF and a composite with 99 vol\% CF content were measured as a function of temperature. The conductive mechanism for the long CF can be modeled by VRH theory. However, for the composite with a $99-$ vol\% short CF content, the calculated temperature-dependent hopping distance $R$ and the average hopping energy $W$ reflected two different mechanisms with a considerable gap at approximately $-120^{\circ} \mathrm{C}$. Thus, $\mathrm{VRH}$ theory is not applicable to composite systems with conductive fillers containing air voids. Consequently, the conductivities of composites with a 99- and 30vol\% CF content were analyzed in terms of the tunneling effect from thermal fluctuations. A nonparabolic potential barrier was used in the theoretical formulation of the conductivity. A simulation was used to select the values of $\lambda$ (a dimensionless parameter given by $\lambda=$ $\left.0.795 e^{2} / 4 D K V_{0}\right), D$ (the potential barrier width) and $A$ (the surface area associated with the tunneling effect) that produced the best fits of the theoretical results to the experimental data. The selected values were limited to a very narrow range. That is, the $D, \lambda$ and $A$ values for the composite with a $99-$ vol $\%$ CF content were $\sim 0.500,0.1201$ and $3.00 \mathrm{~nm}^{2}$, respectively, below $-80{ }^{\circ} \mathrm{C}$. However, the conductivity decreased with temperature above $-80^{\circ} \mathrm{C}$. This behavior was attributed to the decrease in the adhesion power of 1 vol\% UHMWPE to connect adjacent $\mathrm{CFs}$ above $-80^{\circ} \mathrm{C}$, along with the considerable thermal expansion of the air voids between adjacent CFs, which increased the potential barrier width. In contrast, for the composite with a $30-$ vol\% CF content, the CFs were surrounded by UHMWPE layers and the air voids existed as free volumes in the UHMWPE medium. In this case, the free volumes in the matrix were much lower than in pristine UHMWPE. Thus, good agreement between experiment and theory was obtained by choosing suitable values for the $\lambda, D$ and $A$ parameters. The best fits were obtained for $D=1.00 \mathrm{~nm}$, $\lambda=0.080$ and $A=1.64$ to $1.68 \mathrm{~nm}^{2}$ at $51.4 \%$ crystallinity and for $D=1.00 \mathrm{~nm}, \lambda=0.070$ and $A=1.35$ to $1.40 \mathrm{~nm}^{2}$ at $34.6 \%$ crystallinity up to $0{ }^{\circ} \mathrm{C}$. The best fit for the composite with a 25 -vol\% CF content was produced using $D=1.30 \mathrm{~nm}, \lambda=0.055$ and $A=1.25$ to $1.28 \mathrm{~nm}^{2}$. Despite attempts to adjust the parameters, the theoretical 
results deviated slightly from the experimental results for temperatures below $-130{ }^{\circ} \mathrm{C}$ and above $-40{ }^{\circ} \mathrm{C}$. The derivation between the experimental and theoretical results at temperatures $<-130^{\circ} \mathrm{C}$ was attributed to the $\gamma$ relaxation from the motion of the short segments (for example, three or four $\mathrm{CH}_{2}$ groups) in the amorphous phase and the chain ends in the amorphous regions; at temperatures greater than $-40{ }^{\circ} \mathrm{C}$, the deviation between the experimental and theoretical results was attributed to the appearance of a $\beta$ relaxation peak at approximately -25 to $-10^{\circ} \mathrm{C}$, which was associated with the large (macro-Brownian) motion of the amorphous UHMWPE chains and an increase in the amorphous volume. Thus, the fluctuation-induced tunneling effect in the polymer-filler composite systems was used to accurately describe the temperature-dependent conductiviy except over the temperature ranges associated with the $\beta$ and the $\gamma$ relaxations. Note that a nonparabolic potential barrier must be used in the theoretical calculation for the analysis to be valid. The $D$ and $A$ values that were calculated using Equation (1) did not conform to the framework of the tunneling effect. This result was obtained because the temperature dependence of the low-field conductivity was modeled using the variation in $\varepsilon^{*}$, which corresponded to the location of the maximum value of $-\left[\left(T_{1} / T\right) \varepsilon_{\mathrm{T}}^{2}+\left(T_{1} / T_{0}\right) \varphi\left(\varepsilon_{\mathrm{T}}\right)\right]$ in the $0<\varepsilon<1$ interval for polymer-filler composites.

\section{ACKNOWLEDGEMENTS}

We are indebted to Prof. P Sheng of the Hong Kong University of Science and Technology who developed the tunneling effect in many material science areas. He kindly taught us how to derive Equation (14) from Equation (10) in the study by Sheng. ${ }^{19}$

1 Chekanov, Y., Ohnogi, R., Asai, S. \& Sumita, M. Positive temperature coefficient effect of epoxy resin filled with short carbon fibers. Pol. J. 30, 381-387 (1998).

2 Nogales, A., Broza, G., Roslaniec, Z., Schulte, K., Šics, I., Hsiao, B. S., Sanz, A., García-Gutiérrez, M. C., Rueda, D. R., Domingo, C. \& Ezquerra, T. A. Low percolation threshold in nanocomposites based on oxidized single wall carbon nanotubes and poly(butylene terephthalate). Macromolecules 37, 7669-7672 (2004).

3 Connor, M. T., Roy, S., Ezquerra, T. A. \& Calleja, F. J. B. Broadband ac conductivity of conductor-polymer composites. Phys. Rev. B 57, 2286-2294 (1998)

4 Barrau, S., Demont, P., Peigney, A., Laurent, C. \& Lacabanne, C. DC and AC conductivity of carbon nanotubes-polyepoxy composites. Macromolecules 36, 5187 5194 (2003).

5 Zhu, D., Bin, Y. \& Matsuo, M. Electrical conducting behaviors in polymeric composites with carbonaceous fillers. J. Polym. Sci., Part B: Polym. Phys. 45, 1037-1044 (2007).

6 Kono, A., Miyakawa, N., Kawadai, S., Goto, Y., Maruoka, T., Yamamoto, M. \& Horibe, H. Effect of cooling rate after polymer melting on electrical properties of high-density polyethylene/Ni composites. Pol. J. 42, 587-591 (2010).

7 Bello, A., Laredo, E., Marval, J. R., Grimau, M., Arnal, M. L. \& Müller, A. J. Universality and percolation in biodegradable poly( $\varepsilon$-caprolactone)/multiwalled carbon nanotube nanocomposites from broad band alternating and direct current conductivity at various temperatures. Macromolecules 44, 2819-2828 (2011).

8 Bin, Y., Yamanaka, A., Chen, Q., Xi, Y., Jiang, X. \& Matsuo, M. Morphological, electrical and mechanical properties of ultrahigh molecular weight polyethylene and multi-wall carbon nanotube composites prepared in decalin and paraffin. Pol. J. 39, 598-609 (2007).

9 Wu, G., Li, B. \& Jiang, J. Carbon black self-networking induced co-continuity of immiscible polymer blends. Polymer (Guildf) 51, 2077-2083 (2010)

10 Aguilar-Hernández, J. \& Potje-Kamloth, K. Evaluation of the electrical conductivity of polypyrrole polymer composites. J. Phys. D: Appl. Phys. 34, 1700-1711 (2001)

11 Zhang, R., Baxendale, M. \& Peijs, T. Universal resistivity-strain dependence of carbon nanotube/polymer composites. Phys. Rev. B 76, 195433-195437 (2007).

12 Dawson, J. C. \& Adkins, C. J. Conduction mechanisms in carbon-loaded composites. J. Phys.: Condens. Matter 8, 8321-8338 (1996).

13 Mott, N. F. \& Davis., E. A. Electronic Processes in Non-Crystalline Materials. 2nd edn. Ch. 2 pp 7 (Clarendon Press, Oxford, UK, 1979).

14 Okutan, M., Bakan, H. I., Korkmaz, K. \& Yakuphanoglu, F. Variable range hopping conduction and microstructure properties of semiconducting Co-doped $\mathrm{TiO}_{2}$. Physica $B$ 355, 176-181 (2005).

15 Maddison, D. S. \& Tansley, T. L. Variable range hopping in polypyrrole films of a range of conductivities and preparation methods. J. Appl. Phys. 72, 4677-4682 (1992).

16 Singh, R. K., Kumar, A., Agarwal, K., Kumar, M., Singh, H. K., Srivastava, P. \& Singh, R. $\mathrm{DC}$ electrical conduction and morphological behavior of counter anion-governed genesis of electrochemically synthesized polypyrrole films. J. Polym. Sci., Part B: Polym. Phys. 50, 347-360 (2012)

17 Khan, Z. H., Husain, M., Perng, T. P., Salah, N. \& Habib, S. Electrical transport via variable range hopping in an individual multi-wall carbon nanotube. J. Phys.: Condens. Matter 20, 475207-475213 (2008).

18 Simmons, J. G. Generalized formula for the electric tunnel effect between similar electrodes separated by a thin insulating film. J. Appl. Phys. 34, 1793-1803 (1963).

19 Sheng, P. Fluctuation-induced tunneling conduction in disordered materials. Phys. Rev. B 21, 2180-2195 (1980).

20 Sheng, P., Sichel, E. K. \& Gittleman, J. L. Fluctuation-induced tunneling conduction in carbon-polyvinylchloride composites. Phys. Rev. Lett. 40, 1197-1200 (1978).

21 Kono, A., Shimizu, K., Nakano, H., Goto, Y., Kobayashi, Y., Ougizawa, T. \& Horibe, H. Positive-temperature-coefficient effect of electrical resistivity below melting point of poly(vinylidene fluoride) (PVDF) in Ni particle-dispersed PVDF composites. Polymer (Guildf) 53, 1760-1764 (2012)

22 Łuzny, W. \& Bańka, E. Relations between the structure and electric conductivity of polyaniline protonated with camphorsulfonic acid. Macromolecules 33, 425-429 (2000).

23 Chen, R., Bin, Y., Zhang, R., Dong, E., Ougizawa, T., Kuboyama, K. \& Matsuo, M. Positive temperature coefficient effect of polymer-carbon filler composites under selfheating evaluated quantitatively in terms of potential barrier height and width associated with tunnel current. Polymer (Guildf) 53, 5197-5207 (2012).

24 Jung, W. H. Evaluation of Mott's parameters for hopping conduction in $\mathrm{La}_{0.67} \mathrm{Ca}_{0.33} \mathrm{MnO}_{3}$. J. Mater. Sci. Lett. 17, 1317-1319 (1998).

25 Novak, M., Kokanović, I., Babić, D., Baćani, M. \& Tonejc, A. Variable-range-hopping exponents $1 / 2,2 / 5$ and $1 / 4$ in $\mathrm{HCl}$-doped polyaniline. Synth. Met. 159, 649-653 (2009).

26 Entin-Wohlman, O., Gefen, Y. \& Shapira, Y. Variable-range hopping conductivity in granular materials. J. Phys. C: Solid State Phys. 16, 1161-1167 (1983).

27 Yakuphanoglu, F., Aydin, M., Arsu, N. \& Sekerci, M. A small-molecule organic semiconductor. Semiconductors 38, 468-471 (2004).

28 Bin, Y., Kitanaka, M., Zhu, D. \& Matsuo, M. Development of highly oriented polyethylene filled with aligned carbon nanotubes by gelation/crystallization from solutions. Macromolecules 36, 6213-6219 (2003)

29 Xi, Y., Bin, Y., Chiang, C. K. \& Matsuo, M. Dielectric effects on positive temperature coeffcient composites of polyethylene and short carbon fibers. Carbon NY 45 , 1302-1309 (2007).

30 Fizazi, A., Moulton, J., Pakbaz, K., Rughooputh, S. D. D. V., Smith, P. \& Heeger, A. J. Percolation on a self-assembled network: decoration of polyethylene gels with conducting polymer. Phys. Rev. Lett. 64, 2180-2183 (1990).

31 Zhang, J., Mine, M., Zhu, D. \& Matsuo, M. Electrical and dielectric behaviors and their origins in the three-dimensional polyvinyl alcohol/MWCNT composites with low percolation threshold. Carbon NY 47, 1311-1320 (2009).

$32 \mathrm{Ma}$, Y. J., Zhang, Z., Zhou, F., Lu, L., Jin, A. \& Gu, C. Hopping conduction in single ZnO nanowires. Nanotechnology 16, 746-749 (2005).

33 Elimat, Z. M., Zihlif, A. M. \& Ragosta, G. DC electrical conductivity of poly(methyl methacrylate)/carbon black composites at low temperatures. J Mater. Sci.: Mater. Electron 19, 1035-1038 (2008).

34 Yasin, S. F., Zihlif, A. M. \& Ragosta, G. The electrical behavior of laminated conductive polymer composite at low temperature. J. Mater. Sci.: Mater. Electron 16, 63-69 (2005).

35 Stalder, R., Mei, J. G., Subbiah, J., Grand, C., Estrada, L. A., So, F. \& Reynolds, J. R. n-Type conjugated polyisoindigos. Macromolecules 44, 6303-6310 (2011).

36 Klimenko, I. B., Zhuravleva, T. S., Lactionov, A. A. \& Komarova, T. B. Electrophysical properties of ex-rayon and ex-PAN carbon fibers with different temperatures of heattreatment. Mater. Chem. Phys. 31, 319-324 (1992).

37 Sato, M., Ogawa, S., Kaga, T., Sumi, H., Ikenaga, E., Takakuwa, Y., Nihei, M. \& Yokoyama, N. Networked-nanographite wire grown on $\mathrm{SiO}_{2}$ dielectric without catalysts using metal-photoemission-assisted plasma-enhanced CVD. Interconnect Technology Conference (IITC) 6-9 (Burlingame, California, USA, 2010).

38 Madelung, O. Semiconductors: Data Handbook. 3rd edn. Ch. 1.3. (Springer, 2004).

39 Oakey, J., Marr, D. W. M., Schwartz, K. B. \& Wartenberg, M. Influence of polyethylene and carbon black morphology on void formation in conductive composite materials: a SANS study. Macromolecules 32, 5399-5404 (1999).

40 Oakey, J., Marr, D. W. M., Schwartz, B. \& Wartenberg, M. An integrated AFM and SANS approach toward understanding void formation in conductive composite materials. Macromolecules 33, 5198-5203 (2000).

41 Laird, E., Wang, W., Cheng, S., Li, B., Preser, V., Dyatkin, B., Gogotsi, Y. \& Li, C. Y. Polymer single crystal-decorated superhydrophobic buckypaper with controlled wetting and conductivity. ACS Nano. 6, 1204-1213 (2012).

42 Xi, Y., Ishikawa, H., Bin, Y., Chiang, C. K. \& Matsuo, M. Positive temperature coeffcient effect of LMWPE-UHMWPE blends filled with short carbon fibers. Carbon N Y 42, 1699-1706 (2004).

43 Matsuo, M. Ma, L., Azuma, M., He, C \& Suzuki, T. Characteristics of ultradrawn polyethylene films as a function of temperature estimated by the positron annihilation lifetime method. Macromolecules 35, 3059-3065 (2002).

44 Tao, S. J. Positronium Annihilation in molecular substances. J. Chem. Phys. 56, 5499-5508 (1972).

45 Matsuo, M., Bin, Y., Xu, C., Ma, L., Nakaoki, T. \& Suzuki, T. Relaxation mechanism in several kinds of polyethylene estimated by dynamic mechanical measurements, positron annihilation, X-ray and ${ }^{13} \mathrm{C}$ solid-state NMR. Polymer (Guildf) 44, 4325-4340 (2003). 


\section{APPENDIX}

Sheng ${ }^{19}$ formulated the tunneling current density $j(\varepsilon)$ as follows:

$$
\begin{aligned}
& j(\varepsilon)=j_{0}(\varepsilon) \exp [-2 \chi D \xi(\varepsilon)] \varepsilon \leqslant 1 \\
& j_{1}(\varepsilon) \varepsilon>1,
\end{aligned}
$$

where

$$
\begin{aligned}
& j_{0}=\frac{j_{00}}{\eta_{0}^{2}(\varepsilon)} \varepsilon \leqslant 1 \\
& j_{1}(\varepsilon)=j_{00}\left[\frac{1}{\eta_{1}^{2}(\varepsilon)}+9.68\left(\lambda \chi D \overline{\delta_{0}}\right)^{2}(1-\varepsilon)^{2}-4.4\left(\lambda \chi D \overline{\delta_{0}}\right) \frac{1-\varepsilon}{\eta_{1}(\varepsilon)}\right] \varepsilon>1
\end{aligned}
$$

$$
j_{00}=\frac{\pi m e V_{0}^{2}}{4 \chi^{2} D^{2} h^{3}}
$$

where $h$ denotes Planck's constant, $\chi=\left(8 \pi^{2} m V_{0} / h^{2}\right)$ and $j(\varepsilon)$ is required to be continuous at $\varepsilon=1$. The partial conductivity $\Sigma(\varepsilon)$ can be obtained using $j(\varepsilon)$ as follows:

$$
\begin{aligned}
& \sum(\varepsilon)=\frac{1}{\delta_{0}} \frac{d j(\varepsilon)}{d \varepsilon}=\sum_{0}(\varepsilon) \exp [-2 \chi D \xi(\varepsilon)] \varepsilon \leqslant 1 \\
& \sum_{1}(\varepsilon) \varepsilon>1
\end{aligned}
$$

where

$$
\begin{aligned}
\sum_{0}(\varepsilon)= & -\sum_{00} \frac{1}{\eta_{0}^{2}(\varepsilon)}\left[\frac{1}{\eta_{0}(\varepsilon)} \frac{d \eta_{0}(\varepsilon)}{d \varepsilon}+\chi D \frac{d \xi(\varepsilon)}{d \varepsilon}\right] \varepsilon \leqslant 1 \\
\sum_{1}(\delta)= & \sum_{00}\left[\frac{2.2 \lambda \chi D \overline{\delta_{0}}}{\eta_{1}(\varepsilon)}-\frac{1}{\eta_{1}^{3}(\varepsilon)} \frac{d\left(\eta_{1}(\varepsilon)\right)}{d \varepsilon}\right. \\
& \left.+(1-\varepsilon)\left(\frac{2.2 \lambda \chi D \overline{\delta_{0}}}{\eta_{1}^{2}(\varepsilon)} \frac{d \eta_{1}(\varepsilon)}{d \varepsilon}-9.68\left(\lambda \chi D \overline{\delta_{0}}\right)^{2}\right)\right] \varepsilon>1
\end{aligned}
$$

$$
\sum_{00}=\frac{2 j_{00}}{\delta_{0}}
$$

where $\xi(\varepsilon), \eta_{0}(\varepsilon)$ and $\eta_{1}(\varepsilon)$ are given by

$$
\begin{aligned}
& \xi(\varepsilon)=\int_{u_{3}}^{u_{4}}\left(1-\frac{\lambda}{u(1-u)}-\varepsilon \overline{\delta_{0}} u\right)^{1 / 2} d u \\
& \eta_{0}(\varepsilon)=\frac{1}{2} \int_{u_{3}}^{u_{4}} \frac{d u}{\left[1-\frac{\lambda}{u(1-u)}-\varepsilon \overline{\delta_{0}} u\right]^{1 / 2}},
\end{aligned}
$$

where $u_{3}$ and $u_{4}$ denote the two real roots of $V(u, \bar{\delta})$ in Equation (11). In addition,

$$
\eta_{1}(\varepsilon)=\frac{\pi}{2\left(\varepsilon \overline{\delta_{0}}\right)^{1 / 2}}\left[\frac{u^{*}\left(1-u^{*}\right)\left(1-2 u^{*}\right)}{1-3 u^{*}\left(1-u^{*}\right)}\right]^{1 / 2},
$$

where $u^{*}$ denotes the location of $V_{\mathrm{m}}$ such that the following relation is satisfied:

$$
0=\left.\frac{\partial V(u, \varepsilon)}{\partial u}\right|_{u=u^{*}}=\frac{\left(1-2 u^{*}\right)}{(u *)^{2}\left(1-u^{*}\right)^{2}}-\frac{\varepsilon \bar{\delta}_{0}}{\lambda} .
$$

